Review

\title{
Cross-Continental Comparison of National Food Consumption Survey Methods-A Narrative Review
}

Willem De Keyzer ${ }^{1,2, *}$, Tatiana Bracke ${ }^{1}$, Sarah A. McNaughton ${ }^{3}$, Winsome Parnell ${ }^{4}$, Alanna J. Moshfegh ${ }^{5}$, Rosangela A. Pereira ${ }^{6}$, Haeng-Shin Lee ${ }^{7}$, Pieter van't Veer ${ }^{8}$, Stefaan De Henauw ${ }^{1,2}$ and Inge Huybrechts ${ }^{2,9}$

1 Faculty of Science and Technology, Department of Bio- and Food Sciences, University College Ghent-Campus Vesalius, Keramiekstraat 80, B-9000 Ghent, Belgium;

E-Mails: bracketatiana@gmail.com (T.B.); stefaan.dehenauw@ugent.be (S.D.H.)

2 Department of Public Health, Ghent University University Hospital 4K3, De Pintelaan, 185, B-9000 Ghent, Belgium; E-Mail: HuybrechtsI@iarc.fr

3 Centre for Physical Activity and Nutrition Research, School of Exercise and Nutrition Sciences, Deakin University, 221 Burwood Highway, Melbourne, Victoria 3125, Australia;

E-Mail: sarah.mcnaughton@deakin.edu.au

4 Division of Sciences, Department of Human Nutrition, University of Otago, PO Box 56, Dunedin 9054, New Zealand; E-Mail: winsome.parnell@otago.ac.nz

5 Beltsville Human Nutrition Research Center, Agricultural Research Service-USDA, 10300 Baltimore Ave., Beltsville MD 20705, USA;

E-Mail: Alanna.Moshfegh@ars.usda.gov

6 Department of Social Nutrition, Instituto de Nutrição Josué de Castro, Federal University of Rio de Janeiro, Av. Carlos Chagas Filho 373, Cidade Universitária, Ilha do Fundão, Rio de Janeiro 21941902, Brazil; E-Mail: rpereira@uol.com.br

7 Nutrition Management Service and Policy Team, Korea Health Industry Development Institute, Chungbuk 363-700, Korea; E-Mail: haengsin@live.unc.edu

8 Division of Human Nutrition, Wageningen University, Bomenweg 2, Wageningen 6703HD, The Netherlands; E-Mail: Pieter.vantVeer@wur.nl

9 International Agency for Research on Cancer (IARC), 150 Cours Albert Thomas, 69372 Lyon Cedex 08, France

* Author to whom correspondence should be addressed; E-Mail: willem.dekeyzer@hogent.be; Tel.: +32-9-2432372; Fax: +32-9-2201726. 
Abstract: Food consumption surveys are performed in many countries. Comparison of results from those surveys across nations is difficult because of differences in methodological approaches. While consensus about the preferred methodology associated with national food consumption surveys is increasing, no inventory of methodological aspects across continents is available. The aims of the present review are (1) to develop a framework of key methodological elements related to national food consumption surveys, (2) to create an inventory of these properties of surveys performed in the continents NorthAmerica, South-America, Asia and Australasia, and (3) to discuss and compare these methodological properties cross-continentally. A literature search was performed using a fixed set of search terms in different databases. The inventory was completed with all accessible information from all retrieved publications and corresponding authors were requested to provide additional information where missing. Surveys from ten individual countries, originating from four continents are listed in the inventory. The results are presented according to six major aspects of food consumption surveys. The most common dietary intake assessment method used in food consumption surveys worldwide is the 24-HDR (24 h dietary recall), occasionally administered repeatedly, mostly using interview software. Only three countries have incorporated their national food consumption surveys into continuous national health and nutrition examination surveys.

Keywords: nutrition surveys; public health surveillance; nutrition assessment; adult

\section{Introduction}

Food consumption surveys (FCS) are used to estimate intakes of foods and nutrients by a certain target population from a specified region. Usually, they are initiated by governmental organizations to (1) identify deficient or excessive intakes of nutrients, (2) assess accordance with food-based dietary guidelines, or (3) estimate food safety related risks (e.g., contaminant exposures), using national representative samples. However, in light of comparability of results cross-continentally, a thorough overview and comparison of methodological aspects associated with these surveys in each continent is requested and has therefore been initiated in this cross-continental comparison of national food consumption survey methods.

In Europe, efforts have been made to harmonize methodological aspects related to dietary intake assessment (DIA) in the context of national nutrition surveys. Briefly, in the European Food Consumption Survey Method project (EFCOSUM), it was agreed that two non-consecutive 24-HDR (24 h dietary recall), are the most suitable to get internationally comparable data on population means and distributions of actual intake [1]. In addition, the menu-driven standardized 24-HDR program EPIC-Soft (IARC, Lyon, France) was considered to be the most appropriate software for standardized data collection in a pan-European survey. Following the EFCOSUM project, in the European Food Consumption Validation (EFCOVAL) project, EPIC-Soft was upgraded and adapted, and the two non-consecutive 24-HDRs using EPIC-Soft were validated using urinary biomarkers [2]. The software was further evaluated for use in the European Union (EU) Menu project [3], a pan-European food 
consumption survey among EU member states led by EFSA via the feasibility studies EMP-PANEU (Food Consumption Data Collection Methodology for the EU Menu Survey) and PANCAKE (Pilot study for Assessment of Nutrient intake and food Consumption Among Kids in Europe) [4-6]. In 2014, an EFSA report was published aiming to identify and evaluate available European data collection protocols and tools for capturing food consumption information [7]. Previously, Huybrechts et al. reported on the experiences from European national or regional dietary monitoring surveys using the standardized EPIC-Soft program [8], making a further inventory on this standardized methodology used in Europe redundant and leading to the decision to exclude Europe from this cross-continental inventory.

Within the framework of the African Study on Physical Activity and Dietary Assessment Methods (AS-PADAM) project, an inventory questionnaire on the availability of dietary assessment methods was developed and results from eighteen African countries were presented [9]. In contrast to Europe, the inventory showed that for the African continent, high quality, validated and standardized tools are currently lacking, making it difficult to monitor the different phases and speed of the nutrition transition across its countries. Due to this in depth inventory published in the framework of the AS-PADAM project, it was decided to exclude Africa as well from this cross-continental inventory.

As mentioned before, in light of comparability of results cross-continentally, a thorough overview and comparison of methodological aspects associated with these surveys in each continent is requested. Therefore, the aims of the present paper are (1) to develop a framework of key parameters describing methodological aspects of FCS, (2) to create an inventory of methodological properties of national food consumption surveys performed on the continents North-America, South-America, Asia and Australasia, and the remaining continents for which such in depth inventory is still missing, and (3) to discuss and compare these methodological properties cross-continentally.

\section{Experimental Section}

\subsection{Development of the Inventory Framework}

First, key methodological properties of FCS were identified in order to construct a framework available for developing the inventory. This framework was based on the one used by Huybrechts and co-workers [8]. After author debate, it was decided to categorize the properties into six aspects of conducting an FCS: (1) target population, survey design and sampling, (2) dietary intake and other assessments, (3) recruitment of participants, (4) fieldwork characteristics, (5) data/nutrient analyses, and (6) recruitment and training of the interviewers. The framework was designed as a table listing FCS in the rows and property fields in the columns. In total, twenty-nine fields were created. The fields to be completed per survey are presented in Table 1. 
Table 1. Overview of inventory framework.

\begin{tabular}{|c|c|c|}
\hline General items & Recruitment of participants & $\begin{array}{c}\text { Recruitment and training of } \\
\text { interviewers }\end{array}$ \\
\hline Continent & Invitation type & $\begin{array}{c}\text { Recruitment criteria } \\
\text { interviewers }\end{array}$ \\
\hline Country & Incentives & Number of interviewers \\
\hline Survey & Number of participants $(n)$ & $\begin{array}{c}\text { Training material/Training } \\
\text { topics }\end{array}$ \\
\hline $\begin{array}{c}\text { Target population, survey design } \\
\text { and sampling }\end{array}$ & Participation rate $(\%)$ & Training duration \\
\hline Sex & Problems in recruitment & \\
\hline Age (years) & $\begin{array}{c}\text { Fieldwork characteristics and data } \\
\text { controls }\end{array}$ & \\
\hline Sampling method and design & Place of DIA administration & \\
\hline Sampling frame & Time-span fieldwork & \\
\hline $\begin{array}{c}\text { Dietary intake and other } \\
\text { assessments }\end{array}$ & Intermediate controls & \\
\hline Method & Final data controls & \\
\hline Total recalls $(n)$ & Food linking and analysis & \\
\hline Administration & Food classification system & \\
\hline Portion size estimation & Food composition databases & \\
\hline Interview aids/software & $\begin{array}{c}\text { Statistical procedures/ adjustment } \\
\text { (software) }\end{array}$ & \\
\hline Measured anthropometrics & $\begin{array}{l}\text { Methods for calculating under- or } \\
\text { overreporters }\end{array}$ & \\
\hline Biological samples & & \\
\hline
\end{tabular}

DIA: dietary intake assessment.

\subsection{Search Strategy}

As proposed by Blanquer et al. [10], a combined strategy for data acquisition was used. Firstly, a systematic literature search was performed and subsequently, experts were contacted to complete missing information which could not be found in the literature. We used the electronic database MEDLINE (PubMed) and Web of Science to identify studies reporting on food consumption surveys from 1985 to December 2011. Text terms with appropriate truncations, Boolean operators and relevant indexing terms were used. The reference lists in the articles, reviews and textbooks retrieved were also investigated for additional publications yielding a substantial amount of grey literature like reports available on websites of governmental bodies. The key words used in the search were: "national nutrition survey"; "food and nutrition survey"; "dietary consumption survey"; "dietary intake"; "nutrition examination"; "nutrition survey"; and "dietary intake assessment". Additional terms referring to a country or continent were added to this search query for obtaining region-specific information. The selection of continents was based on the seven-continent model excluding Europe (pan-European methodology and inventory of experiences are reported elsewhere [7,8,11]), Africa (availability of dietary assessment tools in Africa have been reported previously by Gavrieli et al. [7]) and Antarctica (no permanent habitation). 
The exclusion criteria that were used to withdraw retrieved surveys were: (1) age (nutrition surveys in children only were excluded given their age-specific approach in terms of dietary intake assessment); (2) indirect or ecological measurement of food intake (e.g., food balance sheets or household budget surveys); (3) absence of dietary intake assessment (e.g., nutritional assessment based on anthropometric or clinical measurements), and (4) publications or reports not available in English and/or not accessible online.

Once the table was completed based on the information available from the retrieved publications, it was e-mailed to principal investigators or corresponding authors of studies reporting on the food consumption survey with an accompanying request to fill in the blanks. This additional information was then merged with the tables and the inventory was distributed to all collaborators for final review.

\section{Results}

The first step of the search strategy yielded a total of 12,605 articles. From this, 4,511 articles met at least one of the exclusion criteria. In the remaining articles, single surveys from individual countries were identified. A total of ten countries from four continents were retained: North-America: Canada, United States (US), Mexico; South-America: Brazil; Asia: China, Japan, Korea (South), Malaysia; Australasia: Australia, New Zealand. In total, data from 28 FCS are presented in the overview.

\subsection{Target Population, Survey Design and Sampling Method}

Table 2 summarizes the study design aspects and methods of the selected surveys. The ages of the target populations ranged from less than 1 year of age to over 80 years. Surveys including all age categories were from Canada, US, Mexico (MHNS-06), China (1991 and onwards), Japan, Korea and Australia. In all surveys, both genders were included except for Mexico (NNS-1999) that included women only. In all surveys, a multistage sampling design was used to select study participants. The sampling frames used for selection of sampling units were based either on census data (US, Mexico, Brazil, Korea and New Zealand), a combination of frames like healthcare registries and labour force data (Canada), strata from counties (China), or enumeration blocks (geographical areas which are artificially created to have about 80 to 120 living quarters (Malaysia)). For Canada, the US, Mexico, China, Korea and Australia the national food consumption survey was also part of a health (examination) survey. The dietary monitoring surveys were cross-sectional, some of which have a continuing character since they are repeated annually or biennially (the US, China, Japan and Korea). For the US and China, participants are included in a cohort for tracking over time.

\subsection{Numbers of Participants and Participation Rates}

In Table 3, recruitment aspects of all selected surveys are listed. Sample sizes of single surveys ranged from 2,596 (Mexico; NNS-1999) to over 30,000 (Canada and Brazil). This latter figure was larger when taking into account the totals of all samples in the continuous programs in the US, China and Korea. Participation rates were above $90 \%$ in Korea (KNHANES 1998) and Malaysia; between $80.0 \%-89.9 \%$ in the US (NHANES 2001, 2005), Mexico (NNS-1999), Brazil, China and Korea; between 70.0\%-79.9\% in Canada, the US (NHANES 2003, 2007 and 2009), and Australia (for the FFQ); and below 70\% in Japan, Australia (for the 24-HDR) and New Zealand. 
Table 2. Target population, survey design and sampling method of national nutrition surveys per continent.

\begin{tabular}{|c|c|c|c|c|c|c|c|}
\hline $\begin{array}{c}\text { Continent } \\
\text { Country } \\
\text { [Ref.] }\end{array}$ & Survey name & Institution & Year(s) & Sex & Age (years) & Sampling method and design & Sampling frame \\
\hline \multicolumn{8}{|c|}{ North-America } \\
\hline $\begin{array}{l}\text { Canada } \\
{[12,13]}\end{array}$ & $\begin{array}{c}\text { Canadian Community } \\
\text { Health Survey - Nutrition } \\
\text { (CCHS) }\end{array}$ & Statistics Canada & 2004 & $\mathrm{M}$ and $\mathrm{F}$ & $\begin{array}{l}\text { All age } \\
\text { categories } \\
(<1-71+)\end{array}$ & $\begin{array}{l}\text { Two-step strategy: } \\
\text { 1) } 80 \text { units in } 14 \text { age/sex groups per } \\
\text { province } \\
\text { 2) power allocation scheme for remaining } \\
\text { anticipated units }\end{array}$ & $\begin{array}{l}4 \text { frames: Labour Force Survey (LFS) area } \\
\text { frame, CCHS } 2.1 \text { dwellings, Prince Edward } \\
\text { Island and Manitoba Healthcare registries }\end{array}$ \\
\hline \multirow[t]{5}{*}{$\begin{array}{c}U S \\
{[14,15]}\end{array}$} & $\begin{array}{l}\text { What we Eat in America } \\
\text { (WWEIA), National } \\
\text { Health and Nutrition } \\
\text { Examination Survey } \\
\text { (Continuous NHANES) }\end{array}$ & $\begin{array}{l}\text { National Center for } \\
\text { Health Statistics } \\
\text { (NCHS) from the } \\
\text { Centers for Disease } \\
\text { Control and } \\
\text { Prevention (CDC) }\end{array}$ & $\begin{array}{c}2001- \\
2002\end{array}$ & $\mathrm{M}$ and $\mathrm{F}$ & $\begin{array}{l}\text { All age } \\
\text { categories } \\
(<1-80+)\end{array}$ & $\begin{array}{l}\text { Stratified, multistage probability sample: } \\
\text { Primary Sampling Units (PSUs) } \\
\text { (counties) }>\text { segments within PSUs } \\
\text { (blocks containing a cluster of } \\
\text { households) }>\text { households within } \\
\text { segments }>\text { one or more participants } \\
\text { within households }\end{array}$ & $\begin{array}{l}\text { PSU samples were selected from a frame of } \\
\text { all U.S. counties, using the } 2000 \text { census } \\
\text { data and associated estimates and } \\
\text { projections }\end{array}$ \\
\hline & & & $\begin{array}{c}2003- \\
2004\end{array}$ & " & $"$ & " & $" 1$ \\
\hline & & & $\begin{array}{c}2005- \\
2006\end{array}$ & $" 1$ & $"$ & $\prime \prime$ & " \\
\hline & & & $\begin{array}{c}2007- \\
2008\end{array}$ & $"$ & $"$ & $"$ & $" 1$ \\
\hline & & & $\begin{array}{c}2009- \\
2010\end{array}$ & $" 1$ & $"$ & $\prime \prime$ & $" 1$ \\
\hline $\begin{array}{l}\text { Mexico } \\
{[16-20]}\end{array}$ & $\begin{array}{c}\text { National Nutrition Survey } \\
1999 \text { (NNS-1999) }\end{array}$ & $\begin{array}{l}\text { Instituto Nacional de } \\
\text { Salud Pública } \\
\text { (INSP) }\end{array}$ & $\begin{array}{c}1998- \\
1999\end{array}$ & $\begin{array}{l}\text { Adolescents } \\
\text { and adults: } \\
\text { F } \\
\text { Children: } \\
\text { M and F }\end{array}$ & $\begin{array}{l}12-49 \\
<12\end{array}$ & $\begin{array}{l}\text { Probabilistic, multistage, stratified cluster } \\
\text { sample: } \\
\text { basic geographical statistical area } \\
(\text { BGSA })>\text { household block }>\text { household }\end{array}$ & $\begin{array}{c}\text { Census data (1995), stratification of BGSA } \\
\text { by socioeconomic status index }\end{array}$ \\
\hline
\end{tabular}


Table 2. Cont.

\begin{tabular}{|c|c|c|c|c|c|c|c|}
\hline & $\begin{array}{c}\text { Encuesta Nacional de } \\
\text { Salud y Nutrición } 2006 \\
\text { (ENSANUT 2006), } \\
\text { Mexican Health and } \\
\text { Nutrition Survey } 2006 \\
\text { (MHNS-06) }\end{array}$ & $\begin{array}{l}\text { Instituto Nacional de } \\
\text { Salud Pública (INSP) }\end{array}$ & $\begin{array}{c}2005- \\
2006\end{array}$ & $\begin{array}{l}\text { Children: } \\
\text { M and } F \\
\text { Adults: } \mathrm{M} \\
\text { and } \mathrm{F}\end{array}$ & $\begin{array}{l}<19 \\
\geq 19\end{array}$ & Multistage, stratified cluster sample & $\mathrm{n} / \mathrm{a}$ \\
\hline \multicolumn{8}{|l|}{ South-America } \\
\hline $\begin{array}{c}\text { Brazil } \\
{[21]}\end{array}$ & $\begin{array}{c}\text { Brazilian Individual } \\
\text { Dietary Survey (IDS 2008- } \\
\text { 2009) }\end{array}$ & $\begin{array}{l}\text { Instituto Brasileiro } \\
\text { de Geografia e } \\
\text { Estatistica (IBGE) }\end{array}$ & $\begin{array}{c}2008- \\
2009\end{array}$ & $\mathrm{M}$ and $\mathrm{F}$ & $\geq 10$ & $\begin{array}{l}\text { Probabilistic two-stage complex cluster } \\
\text { sampling: } \\
\text { census tracts }>\text { households }\end{array}$ & $\begin{array}{c}\text { Census data (2000), a subsample (25\%) of } \\
\text { households selected in the Household } \\
\text { Budget Survey was randomly selected to } \\
\text { participate in the IDS }\end{array}$ \\
\hline \multicolumn{8}{|l|}{ Asia } \\
\hline \multirow[t]{8}{*}{$\begin{array}{l}\text { China } \\
{[22,23]}\end{array}$} & $\begin{array}{l}\text { China Health and Nutrition } \\
\text { Survey (CHNS) }\end{array}$ & $\begin{array}{l}\text { National Institute of } \\
\text { Nutrition and Food } \\
\text { Safety (NINFS) from } \\
\text { the China Center for } \\
\text { Disease Control and } \\
\text { Prevention (CCDC) }\end{array}$ & 1989 & $\begin{array}{l}\text { Children: } \\
\text { M and } \mathrm{F} \\
\text { Adults: } \mathrm{M} \\
\text { and } \mathrm{F}\end{array}$ & $\begin{array}{l}1-6 \\
20-45\end{array}$ & $\begin{array}{l}\text { Multistage, random cluster sample: } \\
\text { province }>\text { county }>\text { PSUs }(n=190)> \\
\text { household }\end{array}$ & $\begin{array}{l}\text { Stratification of counties by income (low, } \\
\text { middle, and high), four counties per } \\
\text { province were selected, PSUs are urban } \\
\text { neighborhoods, suburban neighborhoods, } \\
\text { towns, and rural villages }\end{array}$ \\
\hline & & & 1991 & $\mathrm{M}$ and $\mathrm{F}$ & $\begin{array}{l}\text { All age } \\
\text { categories }\end{array}$ & " & " \\
\hline & & & 1993 & $" 1$ & $" 1$ & " & $" 1$ \\
\hline & & & 1997 & $" 1$ & $" 1$ & " & $" 1$ \\
\hline & & & 2000 & " & " & $\begin{array}{l}\text { Multistage, random cluster sample: } \\
\text { province }>\text { county }>\text { PSUs }(n=216)> \\
\text { household }\end{array}$ & " \\
\hline & & & 2004 & " & " & " & " \\
\hline & & & 2006 & " & " & " & " \\
\hline & & & 2009 & $" 1$ & " & " & $" \prime$ \\
\hline Japan $[24,25]$ & $\begin{array}{l}\text { National Nutrition Survey } \\
\text { in Japan (NNS-J) }\end{array}$ & $\begin{array}{l}\text { National Institute of } \\
\text { Health and Nutrition } \\
\text { (NIHN) }\end{array}$ & $\begin{array}{c}2004- \\
2007\end{array}$ & $\mathrm{M}$ and $\mathrm{F}$ & $\geq 1-70+$ & $\begin{array}{l}\text { Stratified random sample:survey district } \\
\text { units }(n=300)>\text { households }\end{array}$ & $\mathrm{n} / \mathrm{a}$ \\
\hline
\end{tabular}


Table 2. Cont.

\begin{tabular}{|c|c|c|c|c|c|c|c|}
\hline \multirow{7}{*}{$\begin{array}{l}\text { Korea } \\
{[26,27]}\end{array}$} & & Korean Institute for & & & & & \\
\hline & $\begin{array}{l}\text { Korean National Health } \\
\text { and Nutrition Examination } \\
\text { Survey (KNHANES) }\end{array}$ & $\begin{array}{l}\text { Health and Social } \\
\text { Affairs (KIHASA) } \\
\text { and the Korea Health } \\
\text { Industry } \\
\text { Development } \\
\text { Institute (KHIDI) }\end{array}$ & 1998 & $\mathrm{M}$ and $\mathrm{F}$ & $\geq 1-70+$ & $\begin{array}{l}\text { Stratified, multistage probability sample: } \\
\text { PSUs }(n=600)>\text { households }\end{array}$ & Census data, population register \\
\hline & & " & 2001 & $" 1$ & $" 1$ & $" 1$ & " \\
\hline & & $\begin{array}{l}\text { KIHASA, KHIDI } \\
\text { and the Korean } \\
\text { Centers for Disease } \\
\text { Control and } \\
\text { Prevention (KCDC) }\end{array}$ & 2005 & " & " & $" 1$ & $"$ \\
\hline & & $\mathrm{KCDC}$ & 2007 & $" 1$ & $" 1$ & $"$ & $"$ \\
\hline & & $" 1$ & 2008 & $" 1$ & $" 1$ & $" 1$ & $" 1$ \\
\hline & & $" 1$ & 2009 & $" \prime$ & $" 1$ & $" 1$ & $" 1$ \\
\hline $\begin{array}{c}\text { Malaysia } \\
{[28,29]}\end{array}$ & $\begin{array}{l}\text { Malaysian Adult Nutrition } \\
\text { Survey (MANS) }\end{array}$ & $\begin{array}{l}\text { Ministry of Health } \\
\text { Malaysia (MOH-M) }\end{array}$ & 2004 & $\mathrm{M}$ and $\mathrm{F}$ & $18-59$ & $\begin{array}{l}\text { Stratified random sample with } \\
\text { proportional allocation }\end{array}$ & $\begin{array}{c}\text { Enumeration Blocks (EB) and Living } \\
\text { Quarters (LQ) were sampled proportionate } \\
\text { to population size }\end{array}$ \\
\hline \multicolumn{8}{|l|}{ Australasia } \\
\hline $\begin{array}{l}\text { Australia } \\
{[30-33]}\end{array}$ & $\begin{array}{l}\text { National Nutrition Survey } \\
\text { (NNS) }\end{array}$ & $\begin{array}{l}\text { Australian Bureau of } \\
\text { Statistics (ABS) and } \\
\text { Commonwealth } \\
\text { Department of } \\
\text { Health and Family } \\
\text { Services (HFS) }\end{array}$ & 1995 & $\mathrm{M}$ and $\mathrm{F}$ & $\geq 2$ & Multistage, area-based sample & $\begin{array}{l}\text { Householders in private dwellings in } 8 \\
\text { states and territories; Area-based selection } \\
\text { using census collector districts from the } \\
\text { 1991 Population Census }\end{array}$ \\
\hline \multirow[t]{2}{*}{$\begin{array}{c}\text { New Zealand } \\
\text { [34-36] }\end{array}$} & $\begin{array}{l}\text { New Zealand National } \\
\text { Nutrition Survey (NNS97) }\end{array}$ & $\begin{array}{c}\text { New Zealand } \\
\text { Ministry of Health } \\
\text { (MOH-NZ) }\end{array}$ & $\begin{array}{c}1996- \\
1997\end{array}$ & $\mathrm{M}$ and $\mathrm{F}$ & $\geq 15$ & $\begin{array}{c}\text { Multistage, stratified sample: } \\
\text { PSUs }(n=18,000)>\text { households }> \\
\text { participant }\end{array}$ & Area based, census data (1991) \\
\hline & $\begin{array}{c}\text { New Zealand Adult } \\
\text { Nutrition Survey (NZANS) }\end{array}$ & $\prime \prime$ & $\begin{array}{c}2008- \\
2009\end{array}$ & " & $" 1$ & $\begin{array}{l}\text { Multistage, stratified, probability- } \\
\text { proportional-to-size (PPS) sample }\end{array}$ & $\begin{array}{c}\text { Area based, New Zealand census } \\
\text { meshblocks (2006) }\end{array}$ \\
\hline
\end{tabular}


Table 3. Dietary intake and other assessments of national nutrition surveys per continent.

\begin{tabular}{|c|c|c|c|c|c|c|c|c|c|}
\hline \multirow{2}{*}{$\begin{array}{l}\text { Continent } \\
\text { Country } \\
\text { [Ref.] }\end{array}$} & \multirow[b]{2}{*}{ Survey name } & \multirow[b]{2}{*}{ Year(s) } & \multicolumn{5}{|c|}{ Dietary intake assessment } & \multirow[b]{2}{*}{ Measured anthropometrics } & \multirow[b]{2}{*}{ Biological samples } \\
\hline & & & Method & $\begin{array}{l}\text { Total } \\
\text { recalls } \\
(n)\end{array}$ & $\begin{array}{l}\text { Administration } \\
\text { of method }\end{array}$ & Portion size estimation & Interview aids/software & & \\
\hline \multicolumn{10}{|c|}{ North-America } \\
\hline $\begin{array}{l}\text { Canada } \\
{[12,13]}\end{array}$ & $\begin{array}{l}\text { Canadian } \\
\text { Community } \\
\text { Health } \\
\text { Survey - } \\
\text { Nutrition } \\
\text { (CCHS) }\end{array}$ & 2004 & $\begin{array}{l}\text { 24-HDR } \\
\text { (children: 6-11 years } \\
\text { assisted by parents; } \\
<6 \text { years reported by } \\
\text { parents)/ } \\
\text { FFQ (past year, fruit } \\
\text { and vegetables only) }\end{array}$ & $\begin{array}{r}1 \\
\text { (70\% of } \\
\text { sample) } \\
2 \\
\text { (30\% of } \\
\text { sample) }\end{array}$ & $\begin{array}{l}\text { Face-to-face } \\
\text { (first interview) } \\
\text { Telephone } \\
\text { (recall)/ } \\
\text { Paper-pencil }\end{array}$ & $\begin{array}{l}\text { Food model booklet, volume } \\
\text { measures (tablespoon, cup, etc.), } \\
\text { weight measures (ounce, gram, } \\
\text { etc.), dimensions (length, width, } \\
\text { etc.), general measures (relative } \\
\text { sizes, container units) }\end{array}$ & $\begin{array}{l}\text { CAI software, developed by } \\
\text { Statistics Canada (adopted } \\
\text { from AMPM, USDA) }\end{array}$ & Weight and height & $\mathrm{n} / \mathrm{a}$ \\
\hline \multirow[t]{5}{*}{$\begin{array}{c}U S \\
{[14,15]}\end{array}$} & $\begin{array}{l}\text { What we Eat } \\
\text { in America } \\
\text { (WWEIA), } \\
\text { National } \\
\text { Health and } \\
\text { Nutrition } \\
\text { Examination } \\
\text { Survey } \\
\text { (Continuous } \\
\text { NHANES) }\end{array}$ & $\begin{array}{l}2001- \\
2002\end{array}$ & $\begin{array}{l}\text { 24-HDR } \\
\text { (children }<16 \text { years } \\
\text { proxy provided } \\
\text { information)/ } \\
\text { FFQ (past year, } 124 \\
\text { items) }\end{array}$ & 1 & $\begin{array}{l}\text { Face-to-face/ } \\
\text { Paper-pencil }\end{array}$ & $\begin{array}{l}\text { Three-dimensional food models } \\
\text { for first interview. }\end{array}$ & $\begin{array}{l}\text { CAI software, developed by } \\
\text { USDA: Automated Multiple- } \\
\text { Pass Method (AMPM) }\end{array}$ & $\begin{array}{l}\text { Body composition and bone } \\
\text { density (Dual energy x-ray } \\
\text { absorptiometry), body } \\
\text { measurements. }\end{array}$ & $\begin{array}{l}\text { For a complete list of } \\
\text { laboratory components of } \\
\text { NHANES 1999-2012 visit } \\
\text { http://www.cdc.gov/nchs/nh } \\
\text { anes/about_nhanes.htm. }\end{array}$ \\
\hline & & $\begin{array}{l}2003- \\
2004\end{array}$ & $"$ & $\begin{array}{r}2(3-10 \\
\text { day } \\
\text { interval })\end{array}$ & $\begin{array}{l}\text { Face-to-face } \\
\text { (first interview) } \\
\text { Telephone } \\
\text { (recall) }\end{array}$ & $\begin{array}{l}\text { Three-dimensional food models } \\
\text { for first interview. } \\
\text { USDA's Food Model Booklet } \\
\text { (two-dimensional drawings of } \\
\text { glasses, mugs, bowls, mounds, } \\
\text { circles, etc.) and three- } \\
\text { dimensional models (measuring } \\
\text { cups and spoons, a ruler, and two } \\
\text { household spoons) for telephone } \\
\text { interview. }\end{array}$ & " & $\prime \prime$ & " \\
\hline & & $\begin{array}{l}2005- \\
2006\end{array}$ & $"$ & $\prime \prime$ & $"$ & " & " & " & " \\
\hline & & $\begin{array}{l}2007- \\
2008\end{array}$ & " & " & $"$ & " & " & " & " \\
\hline & & $\begin{array}{l}2009- \\
2010\end{array}$ & " & " & " & " & " & " & " \\
\hline
\end{tabular}


Table 3. Cont

\begin{tabular}{|c|c|c|c|c|c|c|c|c|c|}
\hline \multirow[t]{2}{*}{$\begin{array}{l}\text { Mexico } \\
{[16-20]}\end{array}$} & $\begin{array}{l}\text { National } \\
\text { Nutrition } \\
\text { Survey 1999 } \\
\text { (NNS-1999) }\end{array}$ & 1998-1999 & 24-HDR & 1 & $\mathrm{n} / \mathrm{a}$ & $\mathrm{n} / \mathrm{a}$ & $\mathrm{n} / \mathrm{a}$ & $\begin{array}{l}\text { Weight and height (in women, } \\
\text { waist and hip circumferences) }\end{array}$ & $\begin{array}{l}\text { Capillary blood: } \\
\text { concentration of } \\
\text { hemoglobin } \\
\text { Venous blood and urine: } \\
\text { assessment of micronutrient } \\
\text { status }\end{array}$ \\
\hline & $\begin{array}{l}\text { Encuesta } \\
\text { Nacional de } \\
\text { Salud y } \\
\text { Nutrición 2006 } \\
\text { (ENSANUT } \\
\text { 2006), } \\
\text { Mexican } \\
\text { Health and } \\
\text { Nutrition } \\
\text { Survey 2006 } \\
\text { (MHNS-06) }\end{array}$ & 2005-2006 & $\begin{array}{l}\text { Semi- } \\
\text { quantitative } \\
\text { FFQ } \\
\text { (past } 7 \text { days, } \\
101 \text { foods, } 14 \\
\text { food groups) }\end{array}$ & & $\mathrm{n} / \mathrm{a}$ & $\mathrm{n} / \mathrm{a}$ & $\mathrm{n} / \mathrm{a}$ & & \\
\hline \multicolumn{10}{|l|}{$\begin{array}{l}\text { South- } \\
\text { America }\end{array}$} \\
\hline $\begin{array}{l}\text { Brazil } \\
{[21]}\end{array}$ & $\begin{array}{l}\text { Brazilian } \\
\text { Individual } \\
\text { Dietary Survey } \\
\text { (IDS 2008- } \\
\text { 2009) }\end{array}$ & 2008-2009 & $\begin{array}{l}\text { 2-day EDR } \\
\text { (non- } \\
\text { consecutive on } \\
\text { pre-determined } \\
\text { days spanning } \\
\text { one week) }\end{array}$ & & $\begin{array}{l}\text { Paper pencil, face- } \\
\text { to-face interview to } \\
\text { review food records }\end{array}$ & $\begin{array}{l}\text { Picture book (pictures of } \\
\text { plates, glasses, bottles and } \\
\text { cutlery) }\end{array}$ & CAPI software & Weight and height & $\mathrm{n} / \mathrm{a}$ \\
\hline \multicolumn{10}{|l|}{ Asia } \\
\hline \multirow[t]{8}{*}{$\begin{array}{l}\text { China } \\
{[22,23]}\end{array}$} & $\begin{array}{l}\text { China Health } \\
\text { and Nutrition } \\
\text { Survey } \\
\text { (CHNS) }\end{array}$ & 1989 & $\begin{array}{l}24-\mathrm{HDR} \\
\text { (children }<12 \\
\text { years proxy } \\
\text { provided } \\
\text { information) }\end{array}$ & $\begin{array}{r}3 \\
\text { (consecuti } \\
\text { ve on pre- } \\
\text { determine } \\
\text { d days } \\
\text { spanning } \\
\text { one week) }\end{array}$ & $\begin{array}{l}\text { Paper pencil, face- } \\
\text { to-face interview }\end{array}$ & $\begin{array}{l}\text { Food models and picture } \\
\text { aids }\end{array}$ & $\mathrm{n} / \mathrm{a}$ & $\begin{array}{l}\text { Weight and height, head } \\
\text { circumference, arm } \\
\text { circumference, and waist-hip } \\
\text { ratio }\end{array}$ & None \\
\hline & & 1991 & " & " & " & " & " & " & " \\
\hline & & 1993 & $"$ & " & $"$ & " & " & " & " \\
\hline & & 1997 & " & " & $"$ & " & " & " & " \\
\hline & & 2000 & " & $"$ & $"$ & " & " & " & " \\
\hline & & 2004 & " & " & $"$ & " & " & " & " \\
\hline & & 2006 & $"$ & " & $"$ & $"$ & " & $"$ & " \\
\hline & & 2009 & " & " & $"$ & " & " & " & Blood collection \\
\hline
\end{tabular}


Table 3. Cont

\begin{tabular}{|c|c|c|c|c|c|c|c|c|c|}
\hline $\begin{array}{c}\text { Japan } \\
{[24,25]}\end{array}$ & $\begin{array}{l}\text { National } \\
\text { Nutrition } \\
\text { Survey in } \\
\text { Japan (NNS-J) }\end{array}$ & 2004-2007 & $\begin{array}{l}\text { 1- or 3-day } \\
\text { semi-weighed } \\
\text { DR/ } \\
\text { FFQ ( } \geq 20 \\
\text { years/ past } 2 \\
\text { months, } 122 \\
\text { foods and } \\
\text { composite } \\
\text { dishes) }\end{array}$ & & $\begin{array}{l}\text { Paper pencil, face-to- } \\
\text { face interview to } \\
\text { review food records/ } \\
\text { Paper-pencil }\end{array}$ & Kitchen scale & $\mathrm{n} / \mathrm{a}$ & $\begin{array}{l}\text { Weight and height (subjects } \\
\text { aged } 1 \text { year or older), } \\
\text { abdominal circumference } \\
\text { (subjects aged } 6 \text { year or older) }\end{array}$ & $\begin{array}{l}\text { Blood collection (subjects } \\
\text { aged } 20 \text { years or older) }\end{array}$ \\
\hline \multirow[t]{6}{*}{$\begin{array}{r}\text { Korea } \\
{[26,27]}\end{array}$} & $\begin{array}{l}\text { Korean } \\
\text { National } \\
\text { Health and } \\
\text { Nutrition } \\
\text { Examination } \\
\text { Survey } \\
\text { (KNHANES) }\end{array}$ & 1998 & $\begin{array}{l}\text { 24-HDR (in } \\
\text { 200 PSUs)/ } \\
\text { FFQ (past } \\
\text { year, } 109 \text { food } \\
\text { items) }\end{array}$ & 1 & $\begin{array}{l}\text { Face-to-face/ } \\
\text { Paper-pencil }\end{array}$ & $\begin{array}{l}\text { Three-dimensional } \\
\text { food models and a } \\
\text { picture book with } \\
\text { color photographs of } \\
\text { foods }\end{array}$ & $\mathrm{n} / \mathrm{a}$ & Weight and height & Blood and urine collection \\
\hline & & 2001 & " & " & " & " & $\mathrm{n} / \mathrm{a}$ & " & " \\
\hline & & 2005 & " & " & " & " & $\mathrm{n} / \mathrm{a}$ & " & " \\
\hline & & 2007 & " & " & " & " & $\mathrm{n} / \mathrm{a}$ & " & " \\
\hline & & 2008 & $"$ & " & " & " & $\mathrm{n} / \mathrm{a}$ & " & " \\
\hline & & 2009 & $"$ & $"$ & " & " & $\mathrm{n} / \mathrm{a}$ & " & " \\
\hline $\begin{array}{l}\text { Malaysia } \\
{[28,29]}\end{array}$ & $\begin{array}{l}\text { Malaysian } \\
\text { Adult } \\
\text { Nutrition } \\
\text { Survey } \\
\text { (MANS) }\end{array}$ & 2004 & $\begin{array}{l}\text { 24-HDR/ } \\
\text { FFQ (past } \\
\text { year, } 126 \\
\text { foods, } 15 \text { food } \\
\text { groups) }\end{array}$ & 1 & $\begin{array}{l}\text { Face-to-face/ } \\
\text { Paper-pencil }\end{array}$ & $\begin{array}{l}\text { Album of food } \\
\text { pictures and } \\
\text { household measures }\end{array}$ & $\begin{array}{l}\text { Nutritionist Pro }{ }^{\mathrm{TM}} \text { Nutrition } \\
\text { Analysis Software (for data entry) }\end{array}$ & Weight and height & $\mathrm{n} / \mathrm{a}$ \\
\hline \multicolumn{10}{|l|}{ Australasia } \\
\hline $\begin{array}{l}\text { Australia } \\
{[30-33]}\end{array}$ & $\begin{array}{l}\text { National } \\
\text { Nutrition } \\
\text { Survey (NNS) }\end{array}$ & 1995 & $\begin{array}{l}\text { 24-HDR } \\
\text { (children: 2-4 } \\
\text { years reported } \\
\text { by adult; } 5-11 \\
\text { yrs assisted by } \\
\text { adult)/ } \\
\text { FFQ }(\geq 12 \\
\text { years/ past } \\
\text { year, } 107 \\
\text { foods) }\end{array}$ & $\begin{array}{r}1 \text { (90\% of } \\
\text { sample) } \\
2(10 \% \text { of } \\
\text { sample) }\end{array}$ & $\begin{array}{l}\text { Face-to-face/ } \\
\text { Paper-pencil }\end{array}$ & $\begin{array}{l}\text { Measuring cups and } \\
\text { spoons, grids and } \\
\text { ruler }\end{array}$ & $\begin{array}{l}\text { Food instruction booklet with } \\
\text { types of foods and quantities of } \\
15 \text { food groups }\end{array}$ & $\begin{array}{l}\text { Weight and height, waist and } \\
\text { hip circumference }\end{array}$ & $n / a$ \\
\hline
\end{tabular}


Table 3. Cont.

\begin{tabular}{|c|c|c|c|c|c|c|c|c|c|}
\hline \multirow[t]{2}{*}{$\begin{array}{c}\text { New } \\
\text { Zealand } \\
\text { [34-36] }\end{array}$} & $\begin{array}{l}\text { New Zealand } \\
\text { National } \\
\text { Nutrition Survey } \\
\text { (NNS97) }\end{array}$ & $\begin{array}{l}1996- \\
1997\end{array}$ & $\begin{array}{l}\text { 24-HDR/ } \\
\text { FFQ (past year, } 9 \\
\text { food categories) }\end{array}$ & $\begin{array}{r}1 \\
2 \\
(n=695)\end{array}$ & $\begin{array}{l}\text { Face- } \\
\text { to-face/ } \\
\text { Paper- } \\
\text { pencil }\end{array}$ & $\begin{array}{l}\text { Cups, spoons, thickness sticks (thickness of meat, fish, } \\
\text { poultry and cheese), photographs , grids and concentric } \\
\text { circles, balls (to estimate apples and oranges), beans bags (to } \\
\text { describe mashed potato and rice), standard serving sizes of } \\
\text { foods and weights }\end{array}$ & $\begin{array}{l}\text { CAPI software, } \\
\text { LINZ24C } \\
\text { (analogous to } \\
\text { AMPM, USDA) }\end{array}$ & $\begin{array}{l}\text { Weight and height, circumference of } \\
\text { waist, hip and arm, waist-hip ratio, } \\
\text { triceps and subscapular skinfold } \\
\text { thickness, elbow breadth }\end{array}$ & $\begin{array}{l}\text { Non-fasting blood } \\
\text { sample: cellular } \\
\text { evaluation, blood } \\
\text { lipids, iron }\end{array}$ \\
\hline & $\begin{array}{l}\text { New Zealand } \\
\text { Adult Nutrition } \\
\text { Survey (NZANS) }\end{array}$ & $\begin{array}{l}2008- \\
2009\end{array}$ & $\begin{array}{l}\text { 24-HDR/ } \\
\text { dietary habits } \\
\text { questionnaire }\end{array}$ & $\begin{array}{r}1(75 \% \text { of } \\
\text { sample) } \\
2(25 \% \text { of } \\
\text { sample })\end{array}$ & $\begin{array}{l}\text { Face- } \\
\text { to-face/ } \\
\text { Paper- } \\
\text { pencil }\end{array}$ & $\begin{array}{l}\text { Food photographs, shape dimensions, food portion } \\
\text { assessment aids (e.g. dried beans) and packaging } \\
\text { information }\end{array}$ & " & $\begin{array}{l}\text { Weight and height, waist } \\
\text { circumference }\end{array}$ & $\begin{array}{l}\text { Non-fasting blood } \\
\text { sample: } \\
\text { cellular evaluation, } \\
\text { blood lipids, iron, } \\
\text { HbA1c } \\
\text { Spot urine sample: } \\
\text { sodium, potassium, } \\
\text { iodine, creatinine }\end{array}$ \\
\hline
\end{tabular}

" : ditto; n/a: not available; EDR: Estimated dietary record; CAI: computer assisted interview; CAPI: computer assisted personal interview; AMPM: Automated Multiple-Pass Method. 


\subsection{Dietary Intake Assessment Methods}

Most surveys used 24-HDR as the principal DIA method (Table 4). Multiple recalls for all participants were available in the US ( 2 recalls in NHANES 2003 and onwards) and China (3 recalls). In some countries, duplicate recalls were available in a subsample only (Canada, Korea, Australia and New Zealand). A computer-assisted personal interview (CAPI) was performed in the US (NHANES 2001), Malaysia and New Zealand. In Canada and the US (NHANES 2003 and onwards), a CAPI was performed during the first recall and a computer assisted telephone interview (CATI) during the second recall. In the surveys from China and Australia, the 24-HDR was performed with paper and pencil in a face-to-face interview. In Korea, a face-to-face interview was performed, no interview software was reported, and in Mexico, the administration of the 24-HDR was also not reported in the study report. A prospective DIA method was only used in Brazil and Japan (2-day EDR and 1- or 3-day semi-weighed DR respectively). Finally, Mexico (MHNS-06) used only a semi-quantitative FFQ to report on frequencies of intake during the past seven days. An FFQ (formerly called Food Propensity Questionnaire) was also used in addition to a principal DIA method to identify frequencies of consumption and non-consumers of various food groups in Canada, the US, Japan, Korea, Malaysia, Australia and New Zealand (NNS97).

\subsection{Fieldwork Characteristics and Data Controls}

In Table 5, the fieldwork aspects of the nutrition surveys are presented. All surveys reported that at least one interview was conducted when the participant was at home. For surveys with multiple interviews, at least one was conducted at home. Interviews could either be a face-to-face or a telephone interview. In cases where the DIA was a dietary record, interviews were performed to review the participant's records and to check for completeness (Brazil and Japan). Another place for administrating the DIA was at mobile examination centres (MEC) (the US, NHANES). The time-span of the fieldwork was at least one year (all seasons) in Canada, the US, Brazil, Korea (KNHANES 2008 and onwards), Malaysia, Australia and New Zealand.

\subsection{Food Linking and Analysis}

Table 6 summarizes features related to data analyses of the nutrition surveys. Surveys using multiple measures of intake are able to correct for within-person variability. Most surveys used the Nusser method (using Software for Intake Distribution Estimation SIDE or C-SIDE) developed at the Iowa State University (ISU) to calculate distributions of usual intake (Canada, US NHANES 2003, Brazil, Korea and New Zealand). For the US, from NHANES 2005 and onwards, the NCI method developed by the National Cancer Institute was used. Finally, in the Australian survey, an equation by the US National Academy of Science (NAS) was used to adjust for within-person variance [33]. Furthermore, misreporting of energy intake was assessed using either the Goldberg method [37] (EI:BMRest) (the US, Brazil, Malaysia and Australia) or the equations by Black and Cole [38] (Canada). Two surveys indicated that no calculation of misreporting was performed (Korea and New Zealand). 
Table 4. Recruitment of the participants in national nutrition surveys per continent.

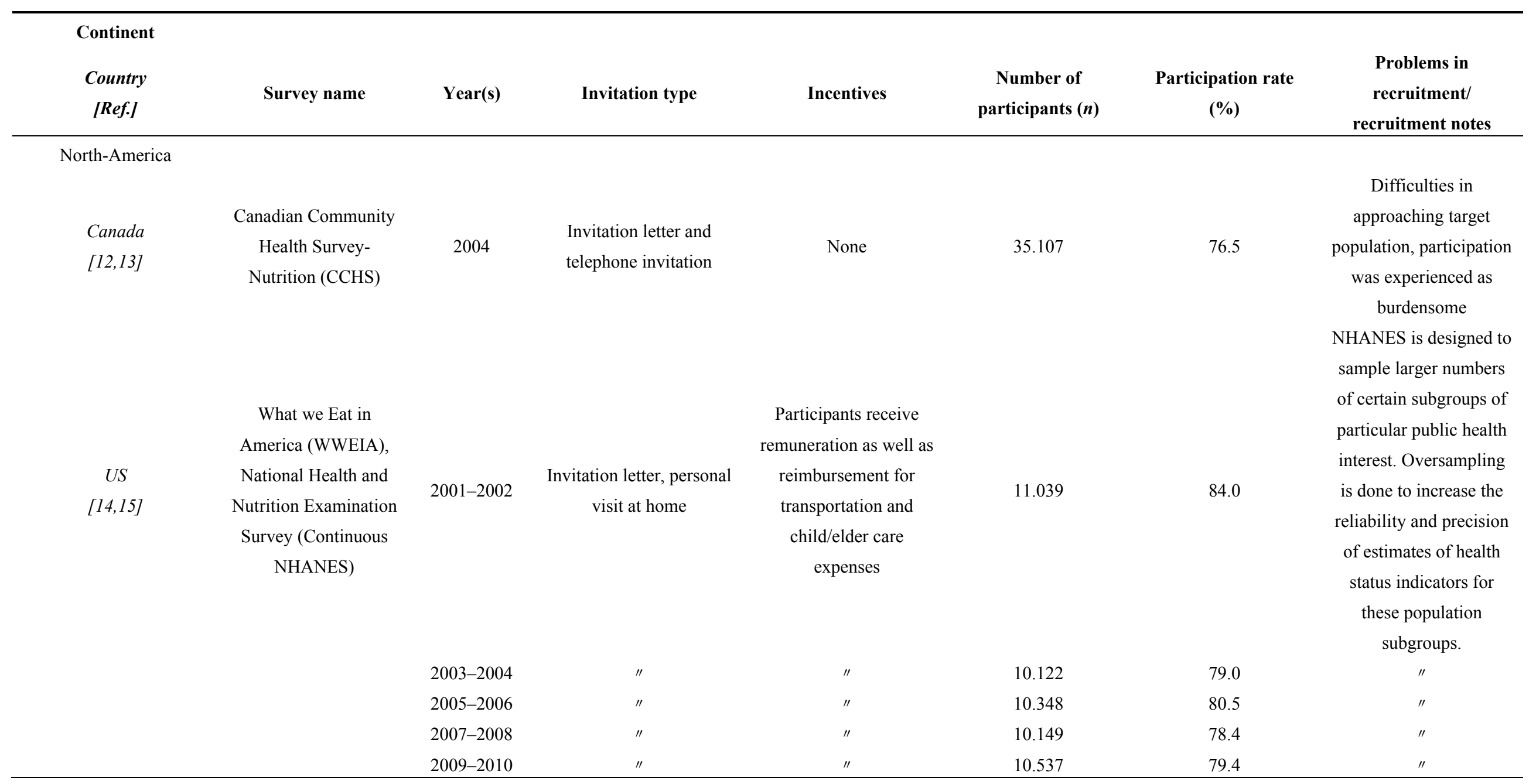


Table 4. Cont.

\begin{tabular}{|c|c|c|c|c|c|c|c|}
\hline \multirow[t]{2}{*}{$\begin{array}{l}\text { Mexico } \\
{[16-20]}\end{array}$} & $\begin{array}{c}\text { National Nutrition } \\
\text { Survey } 1999 \text { (NNS- } \\
\text { 1999) }\end{array}$ & 1998-1999 & $\mathrm{n} / \mathrm{a}$ & $\mathrm{n} / \mathrm{a}$ & $\begin{array}{c}\text { Adolescent F: } 416 \\
\text { Adult F: 2,596 }\end{array}$ & 82.4 & $\mathrm{n} / \mathrm{a}$ \\
\hline & $\begin{array}{c}\text { Encuesta Nacional de } \\
\text { Salud y Nutrición } \\
2006 \text { (ENSANUT } \\
\text { 2006), Mexican Health } \\
\text { and Nutrition Survey } \\
2006 \text { (MHNS-06) }\end{array}$ & 2005-2006 & $\mathrm{n} / \mathrm{a}$ & $\mathrm{n} / \mathrm{a}$ & $\begin{array}{c}\text { Adolescents: 7,464 } \\
\text { Adults: } 21,113\end{array}$ & $\mathrm{n} / \mathrm{a}$ & $\mathrm{n} / \mathrm{a}$ \\
\hline $\begin{array}{c}\text { South-Ameri } \\
\text { Brazil } \\
\text { [21] }\end{array}$ & $\begin{array}{c}\text { Brazilian Individual } \\
\text { Dietary Survey (IDS } \\
\text { 2008-2009) }\end{array}$ & 2008-2009 & Personal visit at home & None & 34.032 & 81.0 & $\begin{array}{c}\text { The burden of } \\
\text { participating in a survey } \\
\text { was reported as a } \\
\text { recruitment problem }\end{array}$ \\
\hline $\begin{array}{l}\text { China } \\
{[22,23]}\end{array}$ & $\begin{array}{c}\text { China Health and } \\
\text { Nutrition Survey } \\
\text { (CHNS) }\end{array}$ & 1989 & Personal visit at home & $\mathrm{n} / \mathrm{a}$ & 15.927 & $\mathrm{n} / \mathrm{a}$ & $\begin{array}{c}\text { Participants leaving in } \\
\text { one survey and moving } \\
\text { back in a later year, } \\
\text { migration of } \\
\text { participants, natural } \\
\text { disasters and major } \\
\text { redevelopment of } \\
\text { housing in all large } \\
\text { urban centres }\end{array}$ \\
\hline & & 1991 & $"$ & $"$ & 14.789 & 88.1 & $" 1$ \\
\hline & & 1993 & $"$ & $"$ & 13.893 & 88.2 & $" 1$ \\
\hline & & 1997 & $" 1$ & $"$ & 15.874 & 80.9 & $"$ \\
\hline & & 2000 & $\prime \prime$ & $\prime \prime$ & 17.054 & 83.0 & $\prime \prime$ \\
\hline
\end{tabular}


Table 4. Cont.

\begin{tabular}{|c|c|c|c|c|c|c|c|}
\hline & & 2004 & $"$ & $"$ & 16.129 & 80.2 & $" 1$ \\
\hline & & 2006 & $"$ & $"$ & 18.764 & 88.0 & $" 1$ \\
\hline & & 2009 & $"$ & $"$ & $\mathrm{n} / \mathrm{a}$ & $\mathrm{n} / \mathrm{a}$ & " \\
\hline $\begin{array}{c}\text { Japan } \\
{[24,25]}\end{array}$ & $\begin{array}{c}\text { National Nutrition } \\
\text { Survey in Japan } \\
\text { (NNS-J) }\end{array}$ & 2004-2007 & $\mathrm{n} / \mathrm{a}$ & $\mathrm{n} / \mathrm{a}$ & $\begin{array}{l}8,762(2004) \\
8,885(2007)\end{array}$ & $\approx 60.0$ (a) & $\mathrm{n} / \mathrm{a}$ \\
\hline \multirow[t]{6}{*}{$\begin{array}{l}\text { Korea } \\
{[26,27]}\end{array}$} & $\begin{array}{c}\text { Korean National } \\
\text { Health and Nutrition } \\
\text { Examination Survey } \\
\text { (KNHANES) }\end{array}$ & 1998 & Invitation letter & Small present & 11.525 & 95.9 & $\mathrm{n} / \mathrm{a}$ \\
\hline & & 2001 & $"$ & $"$ & 10.051 & 81.0 & \\
\hline & & 2005 & $"$ & $\begin{array}{l}\text { Small present and a } \\
\text { letter with individual } \\
\text { results from } \\
\text { examination }\end{array}$ & 9.047 & 80.5 & $\begin{array}{c}\text { The burden of } \\
\text { participating in a survey } \\
\text { and motivation of } \\
\text { participants were } \\
\text { reported as recruitment } \\
\text { problems }\end{array}$ \\
\hline & & 2007 & $" 1$ & $" 1$ & 4.099 & 80.6 & $" 1$ \\
\hline & & 2008 & $"$ & $"$ & 8.641 & 82.0 & $" 1$ \\
\hline & & 2009 & $"$ & $"$ & 9.397 & 82.2 & $" 1$ \\
\hline $\begin{array}{c}\text { Malaysia } \\
{[28,29]}\end{array}$ & $\begin{array}{l}\text { Malaysian Adult } \\
\text { Nutrition Survey } \\
\text { (MANS) }\end{array}$ & 2004 & $\mathrm{n} / \mathrm{a}$ & $\mathrm{n} / \mathrm{a}$ & 6.886 & $\begin{array}{c}93.6(24-\mathrm{HDR}) \\
92.0(\mathrm{FFQ})\end{array}$ & $\mathrm{n} / \mathrm{a}$ \\
\hline
\end{tabular}


Table 4. Cont.

\begin{tabular}{|c|c|c|c|c|c|c|c|}
\hline Australasia & & & & & & & \\
\hline $\begin{array}{l}\text { Australia } \\
\text { [30-33] }\end{array}$ & $\begin{array}{c}\text { National Nutrition } \\
\text { Survey (NNS) }\end{array}$ & 1995 & Invitation letter & None & 13.858 & $\begin{array}{c}61.4 \text { (24-HDR) } \\
76.0 \text { (FFQ) }\end{array}$ & $\mathrm{n} / \mathrm{a}$ \\
\hline \multirow[t]{2}{*}{$\begin{array}{c}\text { New Zealand } \\
\text { [34-36] }\end{array}$} & $\begin{array}{c}\text { New Zealand National } \\
\text { Nutrition Survey } \\
\text { (NNS97) }\end{array}$ & 1996-1997 & $\begin{array}{l}\text { Telephone invitation } \\
\text { and/or personal visit at } \\
\text { home }\end{array}$ & Small present & 4.636 & 50.1 & $\begin{array}{l}\text { Participants of the } \\
\text { Health Survey were } \\
\text { asked if they would } \\
\text { further consent to the } \\
\text { Nutrition Survey which } \\
\text { badly affected the } \\
\text { response rate since } \\
\text { added respondent } \\
\text { burden and time lapse } \\
\text { between both surveys }\end{array}$ \\
\hline & $\begin{array}{l}\text { New Zealand Adult } \\
\text { Nutrition Survey } \\
\text { (NZANS) }\end{array}$ & 2008-2009 & Personal visit at home & $\begin{array}{l}\text { Grocery voucher (if } \\
\text { blood collected) and a } \\
\text { letter with individual } \\
\text { results from } \\
\text { examination }\end{array}$ & 4.721 & 61.0 & " \\
\hline
\end{tabular}


Table 5. Fieldwork characteristics and data controls of national nutrition surveys per continent.

\begin{tabular}{|c|c|c|c|c|c|c|}
\hline $\begin{array}{l}\text { Country } \\
\text { [Ref.] }\end{array}$ & Survey name & Year(s) & Place of DIA administration & $\begin{array}{c}\text { Time- } \\
\text { span } \\
\text { fieldwork }\end{array}$ & Intermediate controls & Final data controls \\
\hline \multicolumn{7}{|c|}{ North-America } \\
\hline $\begin{array}{l}\text { Canada } \\
{[12,13]}\end{array}$ & $\begin{array}{l}\text { Canadian Community Health } \\
\text { Survey-Nutrition (CCHS) }\end{array}$ & 2004 & Participant's home & $\begin{array}{r}\text { Jan } 2004- \\
\text { Jan } 2005\end{array}$ & $\begin{array}{l}\text { Quality control at data entry, checking } \\
\text { completeness and accuracy of collected } \\
\text { data, regular meetings to review the } \\
\text { progress of fieldwork and interviewers. }\end{array}$ & $\begin{array}{l}\text { Identification of extreme values of nutrients } \\
\text { and food groups. Calculation of misreporting } \\
\text { (see table 6). }\end{array}$ \\
\hline \multirow{6}{*}{$\begin{array}{c}\text { US } \\
{[14,15]}\end{array}$} & & & & & & Interview records were reviewed by the \\
\hline & $\begin{array}{l}\text { What we Eat in America } \\
\text { (WWEIA), National Health } \\
\text { and Nutrition Examination } \\
\text { Survey (Continuous } \\
\text { NHANES) }\end{array}$ & $\begin{array}{l}2001- \\
2002\end{array}$ & $\begin{array}{c}\text { First interview: Mobile } \\
\text { Examination Center (MEC) }\end{array}$ & $\begin{array}{l}\text { Jan 2001- } \\
\text { Dec } 2002\end{array}$ & $\begin{array}{l}\text { The CAPI software program has built-in } \\
\text { data edit and consistency checks to reduce } \\
\text { data entry errors. Interviewers were } \\
\text { alerted the when unusual or potentially } \\
\text { erroneous data values were recorded. }\end{array}$ & $\begin{array}{l}\text { NHANES field office staff for accuracy and } \\
\text { completeness. A subset of the household } \\
\text { interviews was verified by re-contacting the } \\
\text { survey participants. Periodically, interviews } \\
\text { were audio-taped and reviewed by NCHS } \\
\text { and contractor staff. }\end{array}$ \\
\hline & & $\begin{array}{c}2003- \\
2004\end{array}$ & $\begin{array}{c}\text { First interview: MEC } \\
\text { Second interview: participant's } \\
\text { home }\end{array}$ & $\begin{array}{l}\text { Jan 2003- } \\
\text { Dec } 2004\end{array}$ & " & $" 1$ \\
\hline & & $\begin{array}{l}2005- \\
2006\end{array}$ & $" 1$ & $\begin{array}{l}\text { Jan 2005- } \\
\text { Dec } 2006\end{array}$ & " & " \\
\hline & & $\begin{array}{l}2007- \\
2008\end{array}$ & " & $\begin{array}{c}\text { Jan } 2007- \\
\text { Dec2008 }\end{array}$ & " & $" \prime$ \\
\hline & & $\begin{array}{c}2009- \\
2010\end{array}$ & " & $\begin{array}{c}\text { Jan 2009- } \\
\text { Dec2010 }\end{array}$ & $" 1$ & " \\
\hline \multirow[t]{2}{*}{$\begin{array}{l}\text { Mexico } \\
{[16-20]}\end{array}$} & $\begin{array}{c}\text { National Nutrition Survey } \\
1999 \text { (NNS-1999) }\end{array}$ & $\begin{array}{c}1998- \\
1999\end{array}$ & $\mathrm{n} / \mathrm{a}$ & $\begin{array}{c}\text { Oct } \\
1998- \\
\text { Mar1999 }\end{array}$ & $\mathrm{n} / \mathrm{a}$ & $\mathrm{n} / \mathrm{a}$ \\
\hline & $\begin{array}{c}\text { Encuesta Nacional de Salud y } \\
\text { Nutrición } 2006 \text { (ENSANUT } \\
\text { 2006), Mexican Health and } \\
\text { Nutrition Survey } 2006 \\
\text { (MHNS-06) }\end{array}$ & $\begin{array}{c}2005- \\
2006\end{array}$ & $\mathrm{n} / \mathrm{a}$ & $\begin{array}{c}\text { Oct } \\
2005- \\
\text { May } 2006\end{array}$ & $\mathrm{n} / \mathrm{a}$ & $\mathrm{n} / \mathrm{a}$ \\
\hline
\end{tabular}


Table 5. Cont.

\begin{tabular}{|c|c|c|c|c|c|c|}
\hline \multicolumn{7}{|c|}{ South-America } \\
\hline $\begin{array}{c}\text { Brazil } \\
{[21]}\end{array}$ & $\begin{array}{l}\text { Brazilian Individual Dietary } \\
\text { Survey (IDS 2008-2009) }\end{array}$ & $\begin{array}{c}2008- \\
2009\end{array}$ & Participant's home & $\begin{array}{c}\text { May } \\
\text { 2008- } \\
\text { May2009 }\end{array}$ & $\begin{array}{l}\text { Cross-check data, quality control during } \\
\text { data entry, completeness and accuracy } \\
\text { checks of collected data, regular meetings } \\
\text { to review the progress of fieldwork and } \\
\text { make adjustments as required }\end{array}$ & Calculation of misreporting (see table 6). \\
\hline \multicolumn{7}{|l|}{ Asia } \\
\hline \multirow[t]{8}{*}{$\begin{array}{l}\text { China } \\
{[22,23]}\end{array}$} & \multirow[t]{8}{*}{$\begin{array}{l}\text { China Health and Nutrition } \\
\text { Survey (CHNS) }\end{array}$} & 1989 & Participant's home & $\mathrm{n} / \mathrm{a}$ & $\begin{array}{l}\text { Internal controls on quality measures have } \\
\text { been based on collecting measures of } \\
\text { selected factors from multiple } \\
\text { perspectives and then using these data to } \\
\text { refine measurements. }\end{array}$ & \multirow[t]{2}{*}{$\begin{array}{l}\text { Individual's average daily dietary intake, } \\
\text { calculated from the household survey, was } \\
\text { compared with dietary intake based on 24-h } \\
\text { recall data. In case of discrepancies, } \\
\text { households were revisited. }\end{array}$} \\
\hline & & 1991 & " & " & $"$ & \\
\hline & & 1993 & $\prime \prime$ & $" 1$ & $\prime \prime$ & " \\
\hline & & 1997 & $\prime \prime$ & $" 1$ & $\prime \prime$ & " \\
\hline & & 2000 & " & " & " & " \\
\hline & & 2004 & " & " & " & $"$ \\
\hline & & 2006 & $\prime \prime$ & $" 1$ & " & $" 1$ \\
\hline & & 2009 & & $" 1$ & $" 1$ & $" 1$ \\
\hline $\begin{array}{l}\text { Japan } \\
{[24,25]}\end{array}$ & $\begin{array}{l}\text { National Nutrition Survey in } \\
\text { Japan (NNS-J) }\end{array}$ & $\begin{array}{c}2004- \\
2007\end{array}$ & Participant's home & $\mathrm{n} / \mathrm{a}$ & $\begin{array}{l}\text { Interview with participant to review food } \\
\text { records and check for completeness }\end{array}$ & $\mathrm{n} / \mathrm{a}$ \\
\hline \multirow[t]{4}{*}{$\begin{array}{c}\text { Korea } \\
{[26,27]}\end{array}$} & \multirow[t]{4}{*}{$\begin{array}{l}\text { Korean National Health and } \\
\text { Nutrition Examination Survey } \\
\text { (KNHANES) }\end{array}$} & 1998 & Participant's home & $\begin{array}{l}\text { Nov } \\
\text { 1998-Dec } \\
1998\end{array}$ & $\begin{array}{c}\text { Cross-check of data, participants were re- } \\
\text { contacted to provide extra information } \\
\text { when the data is incomplete or possibly } \\
\text { wrong }\end{array}$ & $\begin{array}{l}\text { Extreme values for some nutrients and food } \\
\text { groups were calculated }\end{array}$ \\
\hline & & & & Nov & & \\
\hline & & 2001 & $" 1$ & $\begin{array}{l}\text { 2001-Dec } \\
2001 \\
\text { Apr }\end{array}$ & $" 1$ & " \\
\hline & & 2005 & " & $\begin{array}{c}2005- \\
\text { May2005 }\end{array}$ & " & " \\
\hline
\end{tabular}


Table 5. Cont.

\begin{tabular}{|c|c|c|c|c|c|c|}
\hline & & 2007 & $" 1$ & $\begin{array}{l}\text { Jul 2007- } \\
\text { Dec } 2007\end{array}$ & " & " \\
\hline & & 2008 & $" 1$ & $\begin{array}{l}\text { Jan 2008- } \\
\text { Dec } 2008\end{array}$ & " & " \\
\hline & & 2009 & $" 1$ & $\begin{array}{l}\text { Jan 2009- } \\
\text { Dec } 2009\end{array}$ & " & $" 1$ \\
\hline $\begin{array}{c}\text { Malaysia } \\
{[28,29]}\end{array}$ & $\begin{array}{l}\text { Malaysian Adult Nutrition } \\
\text { Survey (MANS) }\end{array}$ & 2004 & Participant's home & $\begin{array}{c}\text { Oct } \\
\text { 2002-Dec } \\
2003\end{array}$ & $\begin{array}{l}\text { Data entry clerks trained to identify, } \\
\text { describe foods and recipes and performed } \\
\text { quality control checks, interviewers } \\
\text { reviewed the recall with the respondent to } \\
\text { check for completeness and accuracy }\end{array}$ & Calculation of misreporting (see Table 6). \\
\hline \multicolumn{7}{|l|}{ Australasia } \\
\hline $\begin{array}{c}\text { Australia } \\
{[30-33]}\end{array}$ & $\begin{array}{l}\text { National Nutrition Survey } \\
\text { (NNS) }\end{array}$ & 1995 & Participant's home & $\begin{array}{c}\text { Feb } \\
1995- \\
\text { Mar } 1996\end{array}$ & $\begin{array}{l}\text { Data was checked immediately after } \\
\text { collection using standardised checklists. } \\
\text { During data entry, all data was scrutinized } \\
\text { and quality control checks for extreme } \\
\text { quantities were built-in to the data entry } \\
\text { computer system. }\end{array}$ & $\begin{array}{l}\text { Extreme values for for energy, macro- } \\
\text { nutrients and micro-nutrients by age and sex } \\
\text { were checked. Calculation of misreporting } \\
\text { (see Table } 6 \text { ). }\end{array}$ \\
\hline \multirow[t]{2}{*}{$\begin{array}{c}\text { New Zealand } \\
\quad[34-36]\end{array}$} & $\begin{array}{c}\text { New Zealand National } \\
\text { Nutrition Survey (NNS97) }\end{array}$ & $\begin{array}{c}1996- \\
1997\end{array}$ & Participant's home & $\begin{array}{c}\text { Dec } \\
1996- \\
\text { Nov } 1997\end{array}$ & $\begin{array}{l}\text { Interviewers sent diet recalls to project } \\
\text { office within } 24 \text { hours of collection so the } \\
\text { project office could check each recall for } \\
\text { accuracy and completeness which enabled } \\
\text { interviewers to go back to participants, } \\
\text { and/or clarify data with project office }\end{array}$ & $\begin{array}{c}\text { Extreme values for nutrient intakes were } \\
\text { scrutinised after conversion of food to } \\
\text { nutrients }\end{array}$ \\
\hline & $\begin{array}{c}\text { New Zealand Adult Nutrition } \\
\text { Survey (NZANS) }\end{array}$ & $\begin{array}{c}2008- \\
2009\end{array}$ & Participant's home & $\begin{array}{c}\text { Oct } \\
2008-\text { Oct } \\
2009\end{array}$ & $"$ & " \\
\hline
\end{tabular}


Table 6. Food linking and analysis of national nutrition surveys per continent.

\begin{tabular}{|c|c|c|c|c|c|c|}
\hline $\begin{array}{l}\text { Continent } \\
\text { Country } \\
\text { [Ref.] }\end{array}$ & Survey name & Year(s) & $\begin{array}{c}\text { Food classification } \\
\text { system }\end{array}$ & Food composition databases & $\begin{array}{c}\text { Statistical } \\
\text { procedures/adjustment } \\
\text { (software) }\end{array}$ & $\begin{array}{c}\text { Methods for } \\
\text { calculating } \\
\text { under- or } \\
\text { overreporting }\end{array}$ \\
\hline \multicolumn{7}{|c|}{ North-America } \\
\hline $\begin{array}{l}\text { Canada } \\
{[12,13]}\end{array}$ & $\begin{array}{c}\text { Canadian Community } \\
\text { Health Survey-Nutrition } \\
\text { (CCHS) }\end{array}$ & 2004 & $\begin{array}{l}\text { Bureau of Nutritional } \\
\text { Sciences (BNS) food } \\
\text { groups, based on } \\
\text { British and American } \\
\text { food group systems }\end{array}$ & Nutrition Survey System (NSS) & $\begin{array}{l}\text { Nusser method using } \\
\text { SIDE (Iowa State } \\
\text { University) }\end{array}$ & $\begin{array}{c}\text { Equations by } \\
\text { Black and Cole }\end{array}$ \\
\hline \multirow[t]{5}{*}{$\begin{array}{c}U S \\
{[14,15]}\end{array}$} & $\begin{array}{l}\text { What we Eat in America } \\
\text { (WWEIA), National } \\
\text { Health and Nutrition } \\
\text { Examination Survey } \\
\text { (Continuous NHANES) }\end{array}$ & $\begin{array}{c}2001- \\
2002\end{array}$ & $\begin{array}{c}\text { Food Surveys } \\
\text { Research Group } \\
\text { (FSRG) defined food } \\
\text { groups }\end{array}$ & USDA Food and Nutrient Database (FNDDS), 1.0 & $\begin{array}{l}\text { SUDAAN was used to } \\
\text { adjust for survey design } \\
\text { effects resulting from } \\
\text { NHANES' complex, } \\
\text { multistage, probability } \\
\text { sampling }\end{array}$ & $\begin{array}{c}\text { Calculation of } \\
\text { EI:BMRest }\end{array}$ \\
\hline & & $\begin{array}{l}2003- \\
2004\end{array}$ & " & USDA Food and Nutrient Database (FNDDS), 2.0 & $\begin{array}{c}\text { Nusser method using C- } \\
\text { SIDE (Iowa State } \\
\text { University) }\end{array}$ & $\prime \prime$ \\
\hline & & $\begin{array}{c}2005- \\
2006\end{array}$ & " & USDA Food and Nutrient Database (FNDDS), 3.0 & NCI method & $" 1$ \\
\hline & & $\begin{array}{c}2007- \\
2008\end{array}$ & " & USDA Food and Nutrient Database (FNDDS), 4.1 & $" 1$ & " \\
\hline & & $\begin{array}{c}2009- \\
2010\end{array}$ & " & USDA Food and Nutrient Database (FNDDS), 5.0 & " & " \\
\hline
\end{tabular}


Table 6. Cont.

\begin{tabular}{|c|c|c|c|c|c|c|}
\hline \multirow[t]{2}{*}{ Mexico [16-20] } & $\begin{array}{c}\text { National Nutrition Survey } \\
1999 \text { (NNS-1999) }\end{array}$ & $\begin{array}{c}1998- \\
1999\end{array}$ & $\mathrm{n} / \mathrm{a}$ & $\begin{array}{l}\text { alimentos para uso en América Latina (PAHO, INCAP), Tablas de } \\
\text { composición de alimentos mexicanos del Instituto Nacional de } \\
\text { Ciencias Médicas y Nutrición Salvador Zubirán, Tablas de valor } \\
\text { nutritivo de los alimentos de mayor consumo en México, Food } \\
\text { composition and nutrition tables (Souci, Fachmann \& Kraut) }\end{array}$ & $\mathrm{n} / \mathrm{a}$ & $\mathrm{n} / \mathrm{a}$ \\
\hline & $\begin{array}{c}\text { Encuesta Nacional de Salud } \\
\text { y Nutrición 2006 } \\
\text { (ENSANUT 2006), } \\
\text { Mexican Health and } \\
\text { Nutrition Survey 2006 } \\
\text { (MHNS-06) }\end{array}$ & $\begin{array}{l}2005- \\
2006\end{array}$ & $\mathrm{n} / \mathrm{a}$ & $\mathrm{n} / \mathrm{a}$ & $\mathrm{n} / \mathrm{a}$ & $\mathrm{n} / \mathrm{a}$ \\
\hline \multicolumn{7}{|l|}{ South-America } \\
\hline $\begin{array}{c}\text { Brazil } \\
{[21]}\end{array}$ & $\begin{array}{l}\text { Brazilian Individual } \\
\text { Dietary Survey (IDS 2008- } \\
\text { 2009) }\end{array}$ & $\begin{array}{l}2008- \\
2009\end{array}$ & $\begin{array}{c}\text { National food } \\
\text { classification system }\end{array}$ & $\begin{array}{c}\text { Nutrition Coordination Center Nutrient Databank (Nutrition Data } \\
\text { System for Research—NDSR, Minneapolis), Brazilian Food } \\
\text { Composition Table (TACO) }\end{array}$ & NCI method & $\begin{array}{c}\text { Calculation of } \\
\text { EI:BMRest }\end{array}$ \\
\hline Asia & & & & & & \\
\hline \multirow[t]{8}{*}{$\begin{array}{l}\text { China } \\
{[22,23]}\end{array}$} & $\begin{array}{c}\text { China Health and Nutrition } \\
\text { Survey (CHNS) }\end{array}$ & 1989 & $\mathrm{n} / \mathrm{a}$ & Food Composition Table for China (ed. 1991) & $\mathrm{n} / \mathrm{a}$ & $\mathrm{n} / \mathrm{a}$ \\
\hline & & 1991 & $" 1$ & " & " & " \\
\hline & & 1993 & " & " & " & " \\
\hline & & 1997 & " & " & " & " \\
\hline & & 2000 & " & " & " & " \\
\hline & & 2004 & " & Food Composition Table for China (ed. 2002) & " & " \\
\hline & & 2006 & $"$ & Food Composition Table for China (ed. 2004) & " & " \\
\hline & & 2009 & $"$ & " & " & $"$ \\
\hline $\begin{array}{l}\text { Japan } \\
{[24,25]}\end{array}$ & $\begin{array}{l}\text { National Nutrition Survey } \\
\text { in Japan (NNS-J) }\end{array}$ & $\begin{array}{l}2004- \\
2007\end{array}$ & $\mathrm{n} / \mathrm{a}$ & Standard Tables of Food Composition in Japan & $\mathrm{n} / \mathrm{a}$ & $\mathrm{n} / \mathrm{a}$ \\
\hline
\end{tabular}

USDA Nutrient database for standard reference, University of

California Food composition database, Tabla de composición de

y Nutrición 2006

on Survey 2006

razilian Individual

\section{Brazil}

[21]

2009)

2009

National food
classification system

utrition Coordination Center Nutrient Databank (Nutrition Data Composition Table (TACO)

Food Composition Table for China (ed. 1991)

Food Composition Table for China (ed. 2002)

Food Composition Table for China (ed. 2004)

Standard Tables of Food Composition in Japan 
Table 6. Cont.

\begin{tabular}{|c|c|c|c|c|c|c|}
\hline \multirow[t]{6}{*}{ Korea $[26,27]$} & $\begin{array}{l}\text { Korean National Health and } \\
\text { Nutrition Examination } \\
\text { Survey (KNHANES) }\end{array}$ & 1998 & $\begin{array}{l}\text { National food } \\
\text { classification system }\end{array}$ & $\begin{array}{l}\text { Food composition table from the National Rural Living Science } \\
\text { Institute }\end{array}$ & $\begin{array}{l}\text { Nusser method } \\
\text { using C-SIDE } \\
\text { (Iowa State } \\
\text { University) }\end{array}$ & Not applied \\
\hline & & 2001 & $" 1$ & $" 1$ & " & " \\
\hline & & 2005 & " & " & " & " \\
\hline & & 2007 & " & " & $" 1$ & " \\
\hline & & 2008 & $" 1$ & $"$ & $" 1$ & $" 1$ \\
\hline & & 2009 & " & $" 1$ & $" 1$ & $" 1$ \\
\hline $\begin{array}{c}\text { Malaysia } \\
{[28,29]}\end{array}$ & $\begin{array}{l}\text { Malaysian Adult Nutrition } \\
\text { Survey (MANS) }\end{array}$ & 2004 & $\mathrm{n} / \mathrm{a}$ & $\begin{array}{c}\text { USDA Food Database, Canadian Food Database, Mexico Food } \\
\text { Database, Malaysian Food Composition Tables (all available in } \\
\text { Nutritionist Pro), Singapore Food Composition Guide, ASEAN } \\
\text { Food Composition Tables, and The China Food Composition } \\
\text { Tables }\end{array}$ & $\mathrm{n} / \mathrm{a}$ & $\begin{array}{l}\text { Calculation of } \\
\text { EI:BMRest }\end{array}$ \\
\hline \multicolumn{7}{|c|}{ - } \\
\hline & & & & & $\begin{array}{l}\text { Adjustment for } \\
\text { within-person } \\
\text { variability using } \\
\text { the equation put }\end{array}$ & \\
\hline $\begin{array}{l}\text { Australia } \\
\text { [30-33] }\end{array}$ & $\begin{array}{l}\text { National Nutrition Survey } \\
\text { (NNS) }\end{array}$ & 1995 & $\begin{array}{l}\text { National food } \\
\text { classification system } \\
\text { developed by ANZFA }\end{array}$ & $\begin{array}{c}\text { NNS nutrient composition database AUSNUT (1999) developed } \\
\text { by the Australia New Zealand Food Authority (ANZFA). Food and } \\
\text { beverage intake data were coded using the Australian Nutrition } \\
\text { Survey System (ANSURS). }\end{array}$ & $\begin{array}{l}\text { forward by the US } \\
\text { National } \\
\text { Academy of } \\
\text { Science (NAS) }\end{array}$ & $\begin{array}{l}\text { Calculation of } \\
\text { EI:BMRest }\end{array}$ \\
\hline & & & & & $\begin{array}{l}\text { Subcommittee on } \\
\text { Criteria for } \\
\text { Dietary } \\
\text { Evaluation (1986) }\end{array}$ & \\
\hline \multirow[t]{2}{*}{$\begin{array}{c}\text { New Zealand } \\
\quad[34-36]\end{array}$} & $\begin{array}{c}\text { New Zealand National } \\
\text { Nutrition Survey (NNS97) }\end{array}$ & $\begin{array}{c}1996- \\
1997\end{array}$ & $\begin{array}{l}\text { National food } \\
\text { classification system }\end{array}$ & $\begin{array}{l}\text { New Zealand Food Composition Database (NZFCD), FOODfiles } \\
\text { electronic subset of data from the NZFCD, NUTTAB Food } \\
\text { Composition Tables (Australia), McCance and Widdowson's } \\
\text { Composition of Foods and other international data as required }\end{array}$ & $\begin{array}{l}\text { Nusser method } \\
\text { using C-SIDE } \\
\text { (Iowa State } \\
\text { University) }\end{array}$ & Not applied \\
\hline & $\begin{array}{c}\text { New Zealand Adult } \\
\text { Nutrition Survey (NZANS) }\end{array}$ & $\begin{array}{c}2008- \\
2009\end{array}$ & " & " & $" 1$ & " \\
\hline
\end{tabular}

" : ditto; n/a: not available. 
Table 7. Recruitment and training of the interviewers in national nutrition surveys per continent.

\begin{tabular}{|c|c|c|c|c|c|c|c|}
\hline $\begin{array}{l}\text { Continent } \\
\text { Country } \\
\text { [Ref.] }\end{array}$ & Survey name & Year(s) & Recruitment criteria interviewers & $\begin{array}{c}\text { Number of } \\
\text { interviewers } \\
(n) \\
\end{array}$ & Training material/Training topics & $\begin{array}{l}\text { Training } \\
\text { duration }\end{array}$ & Remarks \\
\hline \multicolumn{8}{|c|}{ North-America } \\
\hline $\begin{array}{l}\text { Canada } \\
{[12,13]}\end{array}$ & $\begin{array}{c}\text { Canadian Community } \\
\text { Health Survey - Nutrition } \\
\text { (CCHS) }\end{array}$ & 2004 & $\begin{array}{l}\text { Professional interviewers who } \\
\text { work on a variety of surveys, full- } \\
\text { time and part-time }\end{array}$ & 600 & Software training, interview training & 3,5 days & \\
\hline \multirow[t]{5}{*}{$\begin{array}{c}U S \\
{[14,15]}\end{array}$} & $\begin{array}{l}\text { What we Eat in America } \\
\text { (WWEIA), National Health } \\
\text { and Nutrition Examination } \\
\text { Survey (Continuous } \\
\text { NHANES) }\end{array}$ & $\begin{array}{c}2001- \\
2002\end{array}$ & $\begin{array}{l}\text { High School diploma required/BA } \\
\text { preferred }\end{array}$ & $\mathrm{n} / \mathrm{a}$ & $\begin{array}{c}\text { Intensive training course and } \\
\text { supervised practice interviews, } \\
\text { periodic and annual retraining } \\
\text { sessions }\end{array}$ & 2 weeks & \\
\hline & & $\begin{array}{c}2003- \\
2004\end{array}$ & $\prime \prime$ & $\prime \prime$ & $\prime \prime$ & $"$ & \\
\hline & & $\begin{array}{c}2005- \\
2006\end{array}$ & $"$ & $\prime \prime$ & $" 1$ & $"$ & \\
\hline & & $\begin{array}{c}2007- \\
2008\end{array}$ & $"$ & $"$ & $" 1$ & $"$ & \\
\hline & & $\begin{array}{c}2009- \\
2010\end{array}$ & $" 1$ & $\prime \prime$ & $\prime \prime$ & $\prime \prime$ & \\
\hline \multirow[t]{2}{*}{$\begin{array}{l}\text { Mexico } \\
{[16-20]}\end{array}$} & $\begin{array}{c}\text { Mexican Health and } \\
\text { Nutrition Survey } 2006 \\
\text { (MHNS-06) }\end{array}$ & $\begin{array}{c}2005- \\
2006\end{array}$ & $\mathrm{n} / \mathrm{a}$ & $\mathrm{n} / \mathrm{a}$ & $\mathrm{n} / \mathrm{a}$ & $\mathrm{n} / \mathrm{a}$ & \\
\hline & $\begin{array}{l}\text { Encuesta Nacional de Salud } \\
\text { y Nutrición } 2006 \\
\text { (ENSANUT 2006), Mexican } \\
\text { Health and Nutrition Survey } \\
2006 \text { (MHNS-06) }\end{array}$ & $\begin{array}{l}2005- \\
2006\end{array}$ & $\mathrm{n} / \mathrm{a}$ & $\mathrm{n} / \mathrm{a}$ & $\mathrm{n} / \mathrm{a}$ & $\mathrm{n} / \mathrm{a}$ & \\
\hline \multicolumn{8}{|c|}{ South-America } \\
\hline $\begin{array}{c}\text { Brazil } \\
{[21]}\end{array}$ & $\begin{array}{l}\text { Brazilian Individual Dietary } \\
\text { Survey (IDS 2008-2009) }\end{array}$ & $\begin{array}{c}2008- \\
2009\end{array}$ & $\mathrm{n} / \mathrm{a}$ & $\mathrm{n} / \mathrm{a}$ & $\begin{array}{l}\text { Software training, training on } \\
\text { contacting participants, interview } \\
\text { training, data-collection skills }\end{array}$ & 1 week & \\
\hline
\end{tabular}


Table 7. Cont.

\begin{tabular}{|c|c|c|c|c|c|c|c|}
\hline Asia & & & & & & & \\
\hline \multirow[t]{8}{*}{$\begin{array}{l}\text { China } \\
{[22,23]}\end{array}$} & $\begin{array}{c}\text { China Health and Nutrition } \\
\text { Survey (CHNS) }\end{array}$ & 1989 & Trained nutritionists & 160 & $\begin{array}{l}\text { Specific training in the collection of } \\
\text { dietary data for field staff and office } \\
\text { staff }\end{array}$ & 3 days & \\
\hline & & 1991 & $" 1$ & $" 1$ & " & " & \\
\hline & & 1993 & $"$ & $"$ & $" 1$ & $" 1$ & \\
\hline & & 1997 & " & $"$ & $" 1$ & $" 1$ & \\
\hline & & 2000 & $"$ & $"$ & $"$ & $" 1$ & \\
\hline & & 2004 & " & $"$ & $" 1$ & $" 1$ & \\
\hline & & 2006 & $" 1$ & $" 1$ & $" 1$ & $" 1$ & \\
\hline & & 2009 & $"$ & $" 1$ & $" 1$ & " & \\
\hline $\begin{array}{l}\text { Japan } \\
{[24,25]}\end{array}$ & $\begin{array}{c}\text { National Nutrition Survey in } \\
\text { Japan (NNS-J) }\end{array}$ & $\begin{array}{c}2004- \\
2007\end{array}$ & $\begin{array}{l}\text { Registered dietitians and dietitians } \\
\text { for nutrition component of health } \\
\text { survey }\end{array}$ & $\mathrm{n} / \mathrm{a}$ & $\mathrm{n} / \mathrm{a}$ & $\mathrm{n} / \mathrm{a}$ & \\
\hline \multirow[t]{6}{*}{$\begin{array}{l}\text { Korea } \\
{[26,27]}\end{array}$} & $\begin{array}{c}\text { Korean National Health and } \\
\text { Nutrition Examination } \\
\text { Survey (KNHANES) }\end{array}$ & 1998 & Trained dietitians/nutritionists & 160 & $\begin{array}{c}\text { Training on contacting participants, } \\
\text { interview training, data-collection } \\
\text { skills }\end{array}$ & 5 days & \multirow{6}{*}{$\begin{array}{c}\text { A smaller number of well- } \\
\text { trained dietitians were used } \\
\text { after changing to the annual } \\
\text { survey }\end{array}$} \\
\hline & & 2001 & $"$ & 100 & $"$ & 3 days & \\
\hline & & 2005 & $" 1$ & 150 & $" 1$ & 4 days & \\
\hline & & 2007 & $"$ & 10 & $"$ & 11 days & \\
\hline & & 2008 & $" 1$ & 12 & $" 1$ & 10 days & \\
\hline & & 2009 & $" 1$ & 12 & $" 1$ & 15 days & \\
\hline $\begin{array}{c}\text { Malaysia } \\
{[28,29]}\end{array}$ & $\begin{array}{l}\text { Malaysian Adult Nutrition } \\
\text { Survey (MANS) }\end{array}$ & 2004 & $\begin{array}{l}\text { Nutritionists familiar with local } \\
\text { food customs }\end{array}$ & $\mathrm{n} / \mathrm{a}$ & $\begin{array}{l}\text { Training on interviewing and probing } \\
\text { skills, quantification of portion sizes } \\
\text { of foods }\end{array}$ & $\mathrm{n} / \mathrm{a}$ & \\
\hline
\end{tabular}


Table 7. Cont.

\begin{tabular}{|c|c|c|c|c|c|c|c|}
\hline Australasia & & & & & & & \\
\hline $\begin{array}{l}\text { Australia } \\
\text { [30-33] }\end{array}$ & $\begin{array}{l}\text { National Nutrition Survey } \\
\text { (NNS) }\end{array}$ & 1995 & Qualified dietitians and nutritionists & $\mathrm{n} / \mathrm{a}$ & $\begin{array}{l}\text { Intensive training and supervision of } \\
\text { interviewers to reduce non-sampling } \\
\text { errors }\end{array}$ & 2 weeks & \\
\hline $\begin{array}{c}\text { New Zealand } \\
{[34-36]}\end{array}$ & $\begin{array}{c}\text { New Zealand Adult } \\
\text { Nutrition Survey (NZANS) }\end{array}$ & $\begin{array}{c}2008- \\
2009\end{array}$ & $\begin{array}{c}\text { Trained interviewers familiar with } \\
\text { local food customs passing an } \\
\text { admission test }\end{array}$ & $\begin{array}{c}\mathrm{n} / \mathrm{a} \\
\text { (every } \\
\text { interviewer } \\
\text { was assisted } \\
\text { by one } \\
\text { assistant) }\end{array}$ & $\begin{array}{l}\text { Software training, training on } \\
\text { contacting participants, interview } \\
\text { training, data-collection skills and } \\
\text { training on the use of the survey } \\
\text { tools. }\end{array}$ & $\begin{array}{l}\text { Interviewer: } \\
2 \text { weeks } \\
\text { Assistant: } 2 \\
\quad \text { days }\end{array}$ & $\begin{array}{l}\text { Additional training was } \\
\text { provided at the regional level } \\
\text { every two months. Pacific } \\
\text { interviewers and assistants } \\
\text { were trained to survey non- } \\
\text { English speaking Pacific and } \\
\text { Asian immigrant groups. } \\
\text { Additional training was } \\
\text { provided at the regional level } \\
\text { every three months. Pacific } \\
\text { interviewers and assistants } \\
\text { were trained to survey non- } \\
\text { English speaking Pacific and } \\
\text { Asian immigrant groups. }\end{array}$ \\
\hline
\end{tabular}




\subsection{Recruitment and Training of Field Staff}

In Table 7, recruitment and training of the interviewers and field staff in the nutrition surveys are listed. In China, Japan, Korea, Malaysia and Australia, it was mandatory that the interviewers be nutritionists or dietitians. In other countries, interviews were performed by trained interviewers, who were familiar with local food customs (New Zealand), or professional interviewers working on a variety of surveys (Canada). For interviewers in the US, a high school diploma was considered to be the minimum education requirement, as this is necessary for government jobs. Training was provided on a variety of topics like interviewing (and probing) skills (Canada, the US, Brazil, China, Korea, Malaysia, Australia and New Zealand), training on contacting participants, and software training. The duration of these training sessions ranged from three days (China) to fifteen days (Korea, KNHANES 2009). The average duration of reported training programs for interviewers was around seven days.

\section{Discussion}

This review presents an inventory of methodological aspects related to the performance of national food consumption surveys in different continents for which an in depth inventory on the dietary intake assessment methods used was still missing. Inventories covering both standardized and non-standardized data collection protocols and tools for capturing food consumption information on the European and African continent have been published before [7-9]. The present inventory comprises a total of twentyeight food consumption surveys performed in ten countries from four continents: North-America, SouthAmerica, Asia and Australasia. In six countries (Canada, the US, Mexico, China, Korea and Australia), the FCS was part of a larger health examination survey from which three (the US, China and Korea) have been continuous programs. When surveys were not part of a larger health examination survey, the overview shows that questionnaires on health and physical activity were often still included.

The most common approach to assess dietary intake was the use of replicate 24-HDR in combination with an FFQ. In most countries, replicate 24-HDR interviews were administered to subsamples ranging from $<10 \%$ to $30 \%$ of the total sample. For instance, in 2002, the Korean National Nutrition Survey by Season (KNNSS) was conducted and an additional 24-HDR was administered to a subsample of KNHANES over three subsequent seasons to offset seasonal variation in food intake [27]. Duplicate and triplicate 24-HDR were administered to all participants in the US and China respectively. A single 24HDR without additional FFQ was used in Mexico (NNS-1999). In the more recent Mexican Health and Nutrition survey (MHNS-06), the 24-HDR was replaced by a semi-quantitative FFQ that was used to assess frequencies of consumption during the past seven days [17]. This FFQ included the 95\% most consumed foods reported in the 24-HDR collected in the previous survey (MNS-99) [16]. Two countries used a dietary record to assess intakes (Brazil and Japan). However, a research group under the auspices of the Japanese Ministry of Health, Labour and Welfare suggested transferring the method currently in use from a semi-weighed dietary record combined with an FFQ to the 24-HDR making international comparisons possible [25]. Regardless of the DIA methods used, administration took place most often in the participants' homes, providing the major advantage for interviewers to verify food packages or household measures in their home if this could help them to obtain more detailed information. In a study performed by Huybrechts et al. [8], participants of the EFCOVAL project were asked to indicate their 
preferred location for a future 24-HDR interview. Forty-nine percent of the subjects would prefer the study centre (versus $22 \%$ at home and $10 \%$ at work) if the interview was face-to-face and $63 \%$ would prefer to be at home for a telephone interview (compared with $11 \%$ at work). The high number of subjects that preferred the study centre for face-to-face interview might be explained because the EFCOVAL protocol required a visit to the study centre to collect blood samples and to provide participants with material for $24 \mathrm{~h}$ urine collections.

A large variety of portion size estimation tools was used in the different surveys ranging from three-dimensional aids like food models, cups, spoons and thickness sticks to two-dimensional albums or booklets depicting either photographs of foods, plates and glasses, or drawings of glasses, mugs and bowls (United States Department of Agriculture (USDA) food model booklet). The USDA Food Model Booklet was also adapted to create the USDA Food Models for Estimating Portions available for nutrition educators, consumers, and researchers to use outside of the context of the fully computerized Automated Multiple-Pass Method (AMPM) [39]. The AMPM is a validated five-step computerized dietary recall instrument developed by USDA and used in the "What We Eat in America" survey, the dietary intake interview component of the U.S. National Health and Nutrition Examination Survey (NHANES) [40,41]. Computer Assisted Interview (CAI) software is frequently used in national nutrition surveys because it allows structured and standardized collection of dietary intake data. The present overview shows that several countries use USDA-based CAI software and food classification. The leading role of this department is not surprising given its long history that goes back to 1892 [42]. Like North America, Europe has standardized its CAI software for future pan-European food consumption surveys [43]. The EPIC-Soft program, originally developed for the EPIC Study by the International Agency for research on Cancer (IARC), has been validated [44,45] and adapted to fit the purpose of panEuropean food consumption surveys [46]. Recently, a name change of EPIC-Soft to GloboDiet software was announced, since this better suits the current and anticipated use of the increasingly widespread application of the tool worldwide [47].

Given that individual quantitative dietary intake surveys are expensive and difficult to implement, the Food and Agriculture Organization (FAO) Dietary Diversity questionnaire has been developed as a simple proxy to measure access to food at the household level [48] and micronutrient adequacy in women's and children's diets at the individual level $[49,50]$.

Recruitment criteria for interviewers in national nutrition surveys are different between Asia and North America. In all Asian countries presented in the overview and Australia, interviews were conducted by either qualified/registered dietitians or nutritionists. In Japan, no interview was performed since dietary records were used; however, dietitians were recruited for data entry. In Canada and the US, it was not mandatory that the interviewers be dietitians or nutritionists. Both surveys rely either on professional interviewers involved in a variety of surveys or survey staff with a given minimal educational qualification, complemented with specific software and interview training. The duration of the training provided to interviewers varied across all available surveys from 2 days to 15 days (median duration: 7.5 days).

The current overview is the first of its kind to present a wide range of methodological aspects associated with national food consumption surveys across multiple continents. Although substantial efforts have been made to undertake a comprehensive overview, it is inevitable that some surveys were not captured. The present review qualifies as a narrative review and not a systematic review for a number of reasons. During 
the past decades, editors of scientific journals adopted reporting guidelines for producing systematic reviews. This was initiated in the medical research area enabling evidence-based decision making and improved health care. With the advent of these guidelines, publications on randomized (clinical) trials and intervention studies adhere to these criteria for inclusion in future systematic reviews. First, the time window of the present review including studies from 1985 exceeds the initiation of reporting guidelines by a decade so at that time, such guidelines were not yet available. Second, both guidelines for reporting as protocols to perform systematic reviews are not well adopted to studies using observational designs. Just recently, efforts have been made to adapt existing guidelines like the STROBE checklist (STrengthening the Reporting of OBservational studies in Epidemiology) to fit nutritional epidemiology studies (STROBE-NUT, reference equator). Third, a major source for information on methodological aspects of food consumptions surveys like details on sampling, instruments and training of staff are reports, information on websites of public agencies, both qualified as grey literature, and personal communications. These sources of information are sometimes not indexed in scientific databases and are, therefore, difficult to obtain using reproducible search strategies. Therefore, narrative reviews can be criticized because of their limited reproducibility. However, for reasons mentioned before, the twostep approach using both available literature and expert consultation, was the best method available to create the comprehensive overview presented.

This overview shows that the methods used for dietary intake assessment in national nutrition surveys are relatively similar across continents. The most frequently used method is the 24-HDR, sometimes administered repeatedly to correct for within-person variability, and mostly using interview software. Nevertheless, caution is still warranted when comparing results from food surveys between countries because of differences in conversion factors used for calculating nutrients (e.g., energy, protein, etc.). A variety of errors are introduced because many national or regional food composition tables or databases contain incomplete, outdated and unreliable data, or, countries borrow data from publicly available databases and neighbouring countries when such tables or databases are unavailable or inadequate [51].

Notwithstanding the growing consensus about the use of the 24-HDR methodology in food consumption surveys, the assessment remains self-reported. The most accurate and precise method for measuring energy expenditure is the doubly labeled water (DLW) method [52]. In weight stable conditions, one can expect that energy intake equals energy expenditure; hence, DLW is used in studies examining the validity of energy intake assessment. Such validation studies have indicated that the prevalence of energy underreporting in self-reported methods was about 30\% (range: $12 \%-67 \%$ ), and the magnitude of underestimation of energy intake was roughly 15\% (range: 7\%-20\%) [53-55]. These reporting errors vary between men and women and are generally higher among overweight and obese subjects [41].

\section{Conclusions}

The 24-HDR was the most frequently used method in national food consumption surveys worldwide. Although this method is probably the most optimal to monitor dietary intakes of free-living subjects in large samples, it also has limitations and requires in depth training of the interviewers. In addition, future research is still necessary to explore and develop innovative methods that help us to measure dietary intake of populations and subgroups. For national FCS, it is recommended to combine different DIA 
methods like replicate 24-HDR and FFQs. For purposes of comparability of surveys, standardized procedures for data collection are required and a detailed description of the methods used should be included when reporting results. The inventory used in this review can serve as a guide to check if all methodological aspects related to the performance of a FCS are stated in such reports.

\section{Acknowledgments}

The authors gratefully acknowledge the contributions of the Health Statistics Division of Statistics Canada, Simón Barquera, Barry Popkin, Chris Killick-Moran and Shufa Du. The work presented was part of the $\mathrm{PhD}$ dissertation of W.D.K., who was financially supported by the Research Fund of University College Ghent. S.A.M. is funded by an Australian Research Council Future Fellowship (FT100100581).

\section{Author Contributions}

W.D.K. and T.B. performed the search for literature. W.D.K. made the inventory and wrote the manuscript. I.H. and S.D.H. were involved in the conception of the review. All authors reviewed, commented on and approved the final version.

\section{Conflicts of Interest}

The authors declare no conflict of interest.

\section{References}

1. Brussaard, J.H.; Lowik, M.R.; Steingrimsdottir, L.; Moller, A.; Kearney, J.; de Henauw, S.; Becker, W.; Group, E. A European food consumption survey method-Conclusions and recommendations. Eur. J. Clin. Nutr. 2002, 56, S89-S94.

2. de Boer, E.J.; Slimani, N.; van 't Veer, P.; Boeing, H.; Feinberg, M.; Leclercq, C.; Trolle, E.; Amiano, P.; Andersen, L.F.; Freisling, H.; et al. Rationale and methods of the European Food Consumption Validation (EFCOVAL) Project. Eur. J. Clin. Nutr. 2011, 65 (Suppl. 1), S1-S4.

3. EFSA. Declaration of the Advisory Forum on the Pan-European Food Consumption Survey; EFSA: Seville, Spain, 2010.

4. European Food Safety Authority (EFSA). Guidance on the EU Menu methodology. EFSA J. 2014, 12, 77.

5. Ocke, M.; Brants, H.; Dofkova, M.; Freisling, H.; van Rossum, C.; Ruprich, J.; Slimani, N.; Temme, E.; Trolle, E.; Vandevijvere, S.; et al. Feasibility of dietary assessment methods, other tools and procedures for a pan-European food consumption survey among infants, toddlers and children. Eur. J. Nutr. 2014, doi:10.1007/s00394-014-0750-8.

6. Freisling, H.; Ocke, M.C.; Casagrande, C.; Nicolas, G.; Crispim, S.P.; Niekerk, M.; van der Laan, J.; de Boer, E.; Vandevijvere, S.; de Maeyer, M.; et al. Comparison of two food record-based dietary assessment methods for a pan-European food consumption survey among infants, toddlers, and children using data quality indicators. Eur. J. Nutr. 2015, 54, 437-445. 
7. Gavrieli, A.; Naska, A.; Berry, R.; Roe, M.; Harvey, L.; Finglas, P.; Glibetic, M.; Gurinovic, M.; Trichopoulou, A. Dietary Monitoring Tools for Risk Assessment; EFSA supporting publication: Parma, Italy, 2014; p. 287.

8. Huybrechts, I.; Casagrande, C.; Nicolas, G.; Geelen, A.; Crispim, S.P.; de Keyzer, W.; Freisling, H.; de Henauw, S.; de Maeyer, M.; Krems, C.; et al. Inventory of experiences from national/regional dietary monitoring surveys using EPIC-Soft. Eur. J. Clin. Nutr. 2011, 65, S16-S28.

9. Pisa, P.T.; Landais, E.; Margetts, B.; Vorster, H.H.; Friedenreich, C.M.; Huybrechts, I.; Martin-Prevel, Y.; Branca, F.; Lee, W.T.; Leclercq, C.; et al. Inventory on the dietary assessment tools available and needed in Africa: A prerequisite for setting up a common methodological research infrastructure for nutritional surveillance, research and prevention of diet-related non-communicable diseases. Crit. Rev. Food Sci. Nutr. 2014, doi:10.1080/10408398.2014.981630.

10. Blanquer, M.; Garcia-Alvarez, A.; Ribas-Barba, L.; Wijnhoven, T.M.; Tabacchi, G.; Gurinovic, M.; Serra-Majem, L. How to find information on national food and nutrient consumption surveys across Europe: systematic literature review and questionnaires to selected country experts are both good strategies. Br. J. Nutr. 2009, 101, S37-S50.

11. European Food Safety Authority (EFSA). General principles for the collection of national food consumption data in the view of a pan-European dietary survey. EFSA J. 2009, 7, 51.

12. Statistics Canada. Canadian Community Health Survey-Nutrition (CCHS). Available online: http://www.statcan.gc.ca/cgi-bin/imdb/p2SV.pl?Function=getSurvey\&SDDS=5049\&lang=en\&db $=\mathrm{imdb} \& \mathrm{adm}=8 \& \mathrm{dis}=2$ (accessed on 5 November 2012).

13. Health and Statistics Division Canada. Inventory of Food Consumption Surveys-Request for Information. Health and Statistics Division Canada: Ottawa, Canada, 2012.

14. USDA. What We Eat in America, NHANES. Available online: http://www.ars.usda.gov/Services/ docs.htm?docid=13793 (accessed on 24 October 2012).

15. Centers for Disease Control and Prevention. National Health and Nutrition Examination Survey. Available online: http://www.cdc.gov/nchs/nhanes.htm (accessed on 13 October 2012).

16. Barquera, S.; Campirano, F.; Bonvecchio, A.; Hernandez-Barrera, L.; Rivera, J.A.; Popkin, B.M. Caloric beverage consumption patterns in Mexican children. Nutr. J. 2010, 9, 47.

17. Barquera, S.; Hernandez-Barrera, L.; Tolentino, M.L.; Espinosa, J.; Ng, S.W.; Rivera, J.A.; Popkin, B.M. Energy intake from beverages is increasing among Mexican adolescents and adults. J. Nutr. 2008, 138, 2454-2461.

18. Barquera, S.; Rivera, J.A.; Espinosa-Montero, J.; Safdie, M.; Campirano, F.; Monterrubio, E.A. Energy and nutrient consumption in Mexican women 12-49 years of age: Analysis of the National Nutrition Survey 1999. Salud Pública Méx. 2003, 45, 530-539.

19. Resano-Pérez, E.; Méndez-Ramírez, I.; Shamah-Levy, T.; Rivera, J.A.; Sepúlveda-Amor, J. Methods of the National Nutrition Survey, 1999. Salud Pública Méx. 2003, 45, 558-564.

20. Rivera, J.A.; Sepúlveda Amor, J. Conclusions from the Mexican National Nutrition Survey 1999: Translating results into nutrition policy. Salud Pública de Méx. 2003, 45, 565-575.

21. Pereira, R.A.; Duffey, K.J.; Sichieri, R.; Popkin, B.M. Sources of excessive saturated fat, trans fat and sugar consumption in Brazil: an analysis of the first Brazilian nationwide individual dietary survey. Public Health Nutr. 2014, 17, 113-121. 
22. Popkin, B.M.; Du, S.; Zhai, F.; Zhang, B. Cohort Profile: The China Health and Nutrition Survey-Monitoring and understanding socio-economic and health change in China, 1989-2011. Int. J. Epidemiol. 2010, 39, 1435-1440.

23. Chinese Center for Disease Control and Prevention. China Health and Nutrition Survey. Available online: http://www.cpc.unc.edu/projects/china (accessed on 13 November 2012).

24. National Institute of Health and Nutrition Japan. Available online: http://www0.nih.go.jp/ eiken/english/ (accessed on 15 October 2012).

25. Tokudome, S.; Nishi, N.; Tanaka, H. Towards a better National Health and Nutrition Survey in Japan. Lancet 2012, 379, E44.

26. Korea Health Industry Development Institute. Inventory of Food Consumption Surveys-Request for Information. Nutrition Management Service and Policy Team: Chungbuk, Korea, 2012.

27. Kim, D.W.; Shim, J.E.; Paik, H.Y.; Song, W.O.; Joung, H. Nutritional intake of Korean population before and after adjusting for within-individual variations: 2001 Korean National Health and Nutrition Survey Data. Nutr. Res. Pract. 2011, 5, 266-274.

28. Mirnalini, K.; Zalilah, M.; Safiah, M.; Tahir, A.; Siti Haslinda, M.; Siti Rohana, D.; Khairul Zarina, M.; Mohd Hasyami, S.; Normah, H. Energy and Nutrient Intakes: Findings from the Malaysian Adult Nutrition Survey (MANS). Malays. J. Nutr. 2008, 14, 1-24.

29. Norimah, A.; Safiah, M.; Jamal, K.; Siti Haslinda, M.; Zuhaida, H.; Rohida, S.; Fatimah, S.; Siti Norazlin, N.; Poh, B.; Kandiah, M.; et al. Food Consumption Patterns: Findings from the Malaysian Adult Nutrition Survey (MANS). Malays. J. Nutr. 2008, 14, 25-39.

30. Cook, T.; Rutishauser, I.; Seelig, M. Comparable Data on Food and Nutrient Intake and Physical Measurements from the 1983, 1985 and 1995 National Nutrition Surveys; 3057; National Food and Nutrition Monitoring and Surveillance Project - Commonwealth Department of Health and Aged Care: Canberra, Australia, 2001.

31. McLennan, W.; Podger, A. National Nutrition Survey Selected Highlights Australia 1995; Australian Bureau of Statistics Commonwealth Department of Health and Family Services: Canberra, Australia, 1997.

32. Rutishauser, I.H. Getting It Right: How to Use the Data from the 1995 National Nutrition Survey; Commonwealth Department of Health and Aged Care: Canberra, Australia, 2000.

33. Mackerras, D.; Rutishauser, I. 24-Hour national dietary survey data: How do we interpret them most effectively? Public Health Nutr. 2005, 8, 657-665.

34. Ministry of Health. Nutrition Survey (New-Zealand). Available online: http://www.health. govt. nz/nz-health-statistics/national-collections-and-surveys/surveys/current-recent-surveys/nutritionsurvey (accessed on 12 October 2012).

35. Parnell, W.R.; Wilson, N.C.; Russell, D.G. Methodology of the 1997 New Zealand National Nutrition Survey. N. Z. Med. J. 2001, 114, 123-126.

36. Quigley, R.; Watts, C. Food Comes First: Methodologies for the National Nutrition Survey of New Zealand; Public Health Group: Wellington, New Zealand, 1997.

37. Goldberg, G.R.; Black, A.E.; Jebb, S.A.; Cole, T.J.; Murgatroyd, P.R.; Coward, W.A.; Prentice, A.M. Critical evaluation of energy intake data using fundamental principles of energy physiology: 1. Derivation of cut-off limits to identify under-recording. Eur. J. Clin. Nutr. 1991, 45, 569-581. 
38. Black, A.E.; Cole, T.J. Biased over- or under-reporting is characteristic of individuals whether over time or by different assessment methods. J. Am. Diet. Assoc. 2001, 101, 70-80.

39. U.S. Department of Agriculture. Using the USDA Food Models for Estimating Portions; Agricultural Research Service, Beltsville Human Nutrition Research Center, Food Surveys Research Group: Beltsville, MD, USA, 2007.

40. Blanton, C.A.; Moshfegh, A.J.; Baer, D.J.; Kretsch, M.J. The USDA Automated Multiple-Pass Method accurately estimates group total energy and nutrient intake. J. Nutr. 2006, 136, 2594-2599.

41. Moshfegh, A.J.; Rhodes, D.G.; Baer, D.J.; Murayi, T.; Clemens, J.C.; Rumpler, W.V.; Paul, D.R.; Sebastian, R.S.; Kuczynski, K.J.; Ingwersen, L.A.; et al. The US Department of Agriculture Automated Multiple-Pass Method reduces bias in the collection of energy intakes. Am. J. Clin. Nutr. 2008, 88, 324-332.

42. Ahuja, J.K.; Moshfegh, A.J.; Holden, J.M.; Harris, E. USDA food and nutrient databases provide the infrastructure for food and nutrition research, policy, and practice. J. Nutr. 2013, 143, 241S-249S.

43. Ocke, M.C.; Slimani, N.; Brants, H.; Buurma-Rethans, E.; Casagrande, C.; Nicolas, G.; Dofkova, M.; le Donne, C.; Freisling, H.; Geelen, A.; et al. Potential and requirements for a standardized pan-European food consumption survey using the EPIC-Soft software. Eur. J. Clin. Nutr. 2011, 65, S48-S57.

44. Crispim, S.P.; de Vries, J.H.M.; Geelen, A.; Souverein, O.W.; Hulshof, P.J.M.; Lafay, L.; Rousseau, A.-S.; Lillegaard, I.T.L.; Andersen, L.F.; Huybrechts, I.; et al. Two non-consecutive $24 \mathrm{~h}$ recalls using EPIC-Soft software are sufficiently valid for comparing protein and potassium intake between five European centres-Results from the European Food Consumption Validation (EFCOVAL) study. Br. J. Nutr. 2011, 105, 447-458.

45. Crispim, S.P.; Geelen, A.; Souverein, O.W.; Hulshof, P.J.M.; Ruprich, J.; Dofkova, M.; Huybrechts, I.; De Keyzer, W.; Lillegaard, I.T.; Andersen, L.F.; et al. Biomarker-based evaluation of two 24-h recalls for comparing usual fish, fruit and vegetable intakes across European centers in the EFCOVAL Study. Eur. J. Clin. Nutr. 2011, 65, S38-S47.

46. Slimani, N.; Casagrande, C.; Nicolas, G.; Freisling, H.; Huybrechts, I.; Ocke, M.C.; Niekerk, E.M.; van Rossum, C.; Bellemans, M.; De Maeyer, M.; et al. The standardized computerized 24-h dietary recall method EPIC-Soft adapted for pan-European dietary monitoring. Eur. J. Clin. Nutr. 2011, 65, S5-S15.

47. IARC. Formal Announcement of the Name Change from EPIC-Soft ${ }^{\circledR}$ to GloboDiet ${ }^{\circledR}$ Software; Communications Group: Lyon, France, 2014.

48. Hoddinott, J.; Yisehac, Y. Dietary Diversity as a Food Security Indicator. Food and Nutrition Technical Assistance Project (FANTA); Academy for Educational Development (AED): Washington, DC, USA, 2002.

49. Working Group on Infant and Young Child Feeding Indicators. Developing and Validating Simple Indicators of Dietary Quality and Energy Intake of Infants and Young Children in Developing Countries: Summary of Findings from Analysis of 10 Data Sets. Food and Nutrition Technical Assistance (FANTA) Project; Academy for Educational Development (AED): Washington, DC, USA, 2006. 
50. Arimond, M.; Wiesmann, D.; Becquey, E.; Carriquiry, A.; Daniels, M.C.; Deitchler, M.; Fanou-Fogny, N.; Joseph, M.L.; Kennedy, G.; Martin-Prevel, Y.; et al. Simple food group diversity indicators predict micronutrient adequacy of women's diets in 5 diverse, resource-poor settings. $J$. Nutr. 2010, 140, 2059S-2069S.

51. FAO. Food Composition Challenges. Available online: http://www.fao.org/infoods/infoods/foodcomposition-challenges (accessed on 21 May 2013).

52. Schoeller, D.A. Validation of habitual energy intake. Public Health Nutr. 2002, 5, 883-888.

53. Poslusna, K.; Ruprich, J.; de Vries, J.H.; Jakubikova, M.; van't Veer, P. Misreporting of energy and micronutrient intake estimated by food records and 24 hour recalls, control and adjustment methods in practice. Br. J. Nutr. 2009, 101, S73-S85.

54. Hill, R.J.; Davies, P.S. The validity of self-reported energy intake as determined using the doubly labelled water technique. Br. J. Nutr. 2001, 85, 415-430.

55. Trabulsi, J.; Schoeller, D.A. Evaluation of dietary assessment instruments against doubly labeled water, a biomarker of habitual energy intake. Am. J. Physiol. Endocrinol. Metab. 2001, 281, E891-E899.

(C) 2015 by the authors; licensee MDPI, Basel, Switzerland. This article is an open access article distributed under the terms and conditions of the Creative Commons Attribution license (http://creativecommons.org/licenses/by/4.0/). 